\title{
SYMMETRIC CRITICAL KNOTS FOR O'HARA'S ENERGIES
}

\author{
ALEXANDRA GILSBACH AND HEIKO VON DER MOSEL
}

\begin{abstract}
Aвstract. We prove the existence of symmetric critical torus knots for O'Hara's knot energy family $E_{\alpha}, \alpha \in(2,3)$ using Palais' classic principle of symmetric criticality. It turns out that in every torus knot class there are at least two smooth $E_{\alpha}$-critical knots, which supports experimental observations using numerical gradient flows.
\end{abstract}

\section{INTRODUCTION}

Experimenting with R. Scharein's computer program KnotPlot [30] L. H. Kauffman observed in [21] that there might be several distinct local minima present in the presumably complicated knot energy landscape. In particular, a numerical gradient flow implemented in KnotPlot may deform different configurations of the same knot type into distinct final states. For example, the observed shape of the final knot configuration in the torus knot class $\mathcal{T}(2,3)$ heavily depends on whether you start Scharein's flow with a $(2,3)-$ or with a $(3,2)-$ representative; see [21, Section 3]. Moreover, Kauffman reports the presence of a highly symmetrical $(3,4)$-torus knot as the final configuration of that flow that does not yield the absolute minimum of the energy. We have made similar observations using Hermes' numerical gradient flow [20] for integral Menger curvature.

It is the purpose of this paper to support these experimental observations with rigorous analytic results establishing the existence of at least two symmetric critical knots in each torus knot class. Since Kauffman used Scharein's implementation of a Coulomb type selfrepulsion force according to an inverse power of Euclidean distance of different curve points, we focus here on the family of self-repulsive potentials

(1) $\mathrm{E}_{\alpha}(\gamma):=\int_{\mathbb{R} / \mathrm{LZ}} \int_{-\mathrm{L} / 2}^{\mathrm{L} / 2}\left(\frac{1}{|\gamma(\mathrm{u}+w)-\gamma(\mathrm{u})|^{\alpha}}-\frac{1}{\mathrm{~d}_{\gamma}(\mathrm{u}+w, \mathrm{u})^{\alpha}}\right)\left|\gamma^{\prime}(\mathrm{u}+w)\right|\left|\gamma^{\prime}(\mathrm{u})\right| \mathrm{d} w \mathrm{~d} u$

for $\alpha \in[2,3)$, which forms a subfamily of J. O'Hara's energies introduced in [25]. Here, $\gamma: \mathbb{R} /(\mathrm{L} \mathbb{Z}) \rightarrow \mathbb{R}^{3}, \mathrm{~L}>0$, is a Lipschitz continuous closed curve, and

$$
\mathrm{d}_{\gamma}(\mathfrak{u}+\mathfrak{w}, \mathfrak{u}):=\min \left\{\mathscr{L}\left(\left.\gamma\right|_{[\mathfrak{u}, \mathfrak{u}+w]}\right), \mathscr{L}(\gamma)-\mathscr{L}\left(\left.\gamma\right|_{[\mathfrak{u}, \mathfrak{u}+w]}\right)\right\} \text { for }|w| \leqslant \mathrm{L} / 2
$$

denotes the intrinsic distance, i.e., the length of the shorter arc on $\gamma$ connecting the points $\gamma(u)$ with $\gamma(u+w)$. Here, the letter $\mathscr{L}$ denotes the length of a curve.

Remark 1.1. 1. For $\alpha=2$ the energy $\mathrm{E}_{2}$ is called Möbius energy because of its invariance under Möbius transformations; see [15, Theorem 2.1]. For arbitrary $\alpha \in[2,3)$ one still has invariance under isometries in $\mathbb{R}^{3}$ and under reparametrizations.

Date: April 10, 2020.

1991 Mathematics Subject Classification. 49Q10, 53A04, 57M25.

Key words and phrases. knot energy, symmetric criticality, torus knots. 
2. $E_{2}$ can be minimized in arbitrary prescribed prime knot classes according to Freedman, He, and Wang [15, Theorem 4.3], whereas $\mathrm{E}_{\alpha}$ for $\alpha \in(2,3)$ is minimizable in every given tame knot class as shown by O'Hara in [26, Theorem 3.2].

3 . For all $\alpha \in[2,3)$ the once-covered circle uniquely minimizes the energy $E_{\alpha}$, which was shown by Abrams et al. in [1].

For the scaling-invariant version

$$
\mathrm{S}_{\alpha}:=\mathscr{L}^{\alpha-2} \cdot \mathrm{E}_{\alpha}
$$

we prove the following central result.

Theorem 1.2. Let $\mathrm{a}, \mathrm{b} \in \mathbb{Z} \backslash\{0, \pm 1\}$ be relatively prime, $\alpha \in(2,3)$. Then there are at least two arclength parametrized, embedded $S_{\alpha}$-critical curves $\Gamma_{1}, \Gamma_{2} \in C^{\infty}\left(\mathbb{R} / \mathbb{Z}, \mathbb{R}^{3}\right)$ both representing the torus knot class $\mathcal{T}(\mathrm{a}, \mathrm{b})$, such that there is no isometry $\mathrm{I}: \mathbb{R}^{3} \rightarrow \mathbb{R}^{3}$ with $\mathrm{I} \circ \Gamma_{1}(\mathbb{R} / \mathbb{Z})=\Gamma_{2}(\mathbb{R} / \mathbb{Z})$.

In consequence, the gradient flow for $S_{\alpha}$ (or the flow for a linear combination of $E_{\alpha}$ and length $\mathscr{L}$ treated analytically by S. Blatt [5]) might very well get stuck in one of these critical points without having reached the absolute energy minimum. Theorem 1.2 could explain some of the experimental effects described above - in particular those displaying symmetric non-minimizing final configurations since we use discrete rotational symmetries to construct $\Gamma_{1}$ and $\Gamma_{2}$. However, Theorem 1.2 contains no statement about stability, so these $S_{\alpha}$-critical knots may be local minima or merely saddle points.

In contrast to the work of J. Cantarella et al. [10] on symmetric criticality for the nonsmooth ropelength functional we obtain here smooth critical points of the continuously differentiable energy functional $S_{\alpha}$ since we can apply the classic principle of symmetric criticality made rigorous by R. Palais in [27]. This principle can also be applied to various types of geometric curvature energies such as integral Menger curvature or tangent-point energies investigated in [32-34], to produce symmetric critical knots in any knot class that possesses at least one symmetric representative. Suitably scaled versions of those energies do converge to ropelength in the $\Gamma$-limit sense as their integrability exponents tend to infinity. This implies, in particular, that the symmetric critical knots we produce by Palais' principle converge to symmetric ropelength-critical knots; see [16, 17]. At this point, however, it is not clear if we thus obtain in the $\Gamma$-limit the same ropelength-critical points as the ones Cantarella et al. provide in [10].

The Möbius energy, i.e., the case $\alpha=2$, is excluded in Theorem [1.2, in ongoing work [6] we treat this technically more challenging energy. D. Kim and R. Kusner, however, have chosen in [22] a different, in a sense one-dimensional approach to symmetric criticality for the Möbius energy. They restrict their search to torus knots that actually lie on the surfaces of tori foliating the $\mathbb{S}^{3}$ through variations of the tori's radius ratio. It would be interesting to investigate the relation between their Möbius-critical torus knots and the ones we aim for in [6]. Kim and Kusner conjecture in [22, p. 2] on the basis of their numerical experiments with Brakke's evolver [8] that stability of Möbius critical torus knots in $\mathcal{T}(a, b)$ should only be expected when $a=2$ or $b=2$. Stability for symmetric critical knots is still an open problem not only for the scaled O'Hara energies $S_{\alpha}$ but also for all other knot energies mentioned so far.

Let us briefly outline the structure of the paper. In Section 2 we recall the relevant aspects of Palais' principle of symmetric criticality on Banach manifolds. The most important properties of O'Hara's energies $E_{\alpha}$ are presented in Section 3 , such as self-avoidance 
(Lemma 3.1), semicontinuity (Lemma 3.5), and Blatt's characterization [4] of energy spaces (Theorem 3.2) in terms of fractional Sobolev spaces, so-called Sobolev-Slobodetckij spaces. This characterization is crucial in Section 4 to identify the correct Banach manifold (Corollary 4.2), on which Palais' principle of symmetric criticality is applicable. Then we describe discrete rotational symmetries of parametrized curves in terms of a group action of the cyclic group (Definition 4.3 and Lemma 4.4). After checking the effects of reparametrizations on symmetry properties (Corollary 4.7) we focus on the torus knot classes $\mathcal{T}(a, b)$ to find symmetric representatives (Lemma 4.8), and use a direct method in the calculus of variations to minimize $S_{\alpha}$ in symmetric subsets (Theorem 4.9). Using well-known knot theoretic periodicity properties of $\mathcal{T}(a, b)$, we can finally identify two geometrically different symmetric critical knots, which establishes Theorem 1.2. This proof is based on a general result on possible rotational symmetries for general tame knots (Theorem 4.12), for which we present a purely geometric proof, and which may be of independent interest. Some technical intermediate results, e.g. on the Sobolev-Slobodetckij seminorm, or on sets invariant under discrete rotations, are proven in the appendix.

The paper is essentially self-contained not only for the convenience of the reader but also because in places we needed somewhat more refined versions of known results such as Theorem 3.2 .

\section{The PRINCIPLE OF SYMMETRIC CRITICALITY}

In this section we briefly recall the notion of a group action on a in general infinite dimensional Banach manifold in order to formulate a version of Palais' principle of symmetric criticality suitable for our application.

Definition 2.1. Let $k \in \mathbb{N} \cup\{0\}$ and $\mathscr{B}$ a Banach space. Then a Hausdorff space $\mathscr{M}$ is a Banach manifold modeled over $\mathscr{B}$ of class $C^{k}$, or in short, a $C^{k}$-manifold over $\mathscr{B}$ if and only if the following two conditions hold:

(i) For all $x \in \mathscr{M}$ there is an open set $\mathrm{V}_{x} \subset \mathscr{M}$ containing $\mathrm{x}$, and some open set $\Omega_{x} \subset \mathscr{B}$ containing 0 , and a homeomorphism $\phi_{x}: \Omega_{x} \rightarrow V_{x}$ with $\phi_{x}(0)=x$.

(ii) For two distinct points $x, y \in \mathscr{M}$ with $x, y \in V_{x} \cap V_{y}$, the corresponding homeomorphisms $\phi_{x}: \Omega_{x} \rightarrow V_{x} \subset \mathscr{M}$ and $\phi_{y}: \Omega_{y} \rightarrow V_{y} \subset \mathscr{M}$ satisfy

$$
\left.\phi_{y}^{-1} \circ \phi_{x}\right|_{\Omega_{x} \cap \Omega_{y}} \in C^{k}\left(\Omega_{x} \cap \Omega_{y}, \mathscr{B}\right) \text {. }
$$

$\mathscr{M}$ is a smooth, or $\mathrm{C}^{\infty}$-manifold over $\mathscr{B}$ if $\mathscr{M}$ is a $C^{k}$-manifold over $\mathscr{B}$ for all $k \in \mathbb{N}$. The maps $\phi_{x}$ are called local parametrizations, and their inverse mappings $\phi_{\chi}^{-1}: \mathrm{V}_{x} \rightarrow \Omega_{\chi}$ are the local charts. The collection of all charts together with their respective domains forms a $C^{\mathrm{k}}$-atlas of the Banach manifold $\mathscr{M}$.

Example 2.2. Every open subset $\Omega \subset \mathscr{B}$ of a Banach space $\mathscr{B}$ is a smooth manifold over $\mathscr{B}$, since for every $x \in \Omega$ one may choose the parametrization $\phi_{x}:=\operatorname{Id} d_{\mathscr{B}}$, so that the atlas of this simple Banach manifold contains only one element, namely $\left(\operatorname{Id}_{\mathscr{B}}, \Omega\right)$.

In order to incorporate symmetry in a mathematically rigorous way, one uses groups and their action on Banach manifolds; cf. [27, pp. 19,20].

Definition 2.3. Let $(\mathrm{G}, \circ)$ be a group, $\mathscr{B}$ a Banach space, and $\mathscr{M}$ a $\mathrm{C}^{\mathrm{k}}$-manifold over $\mathscr{B}$ for some $k \in \mathbb{N}$. 
(i) $\mathrm{G}$ acts on $\mathscr{M}$ if and only if there is a mapping $\tau: \mathrm{G} \times \mathscr{M} \rightarrow \mathscr{M}$ mapping a pair $(g, x)$ to a point $\tau_{g}(x) \in \mathscr{M}$, such that

$$
\tau_{\mathrm{g} \circ \mathrm{h}}(\mathrm{x})=\tau_{\mathrm{g}}\left(\tau_{\mathrm{h}}(\mathrm{x})\right) \text { for all } \mathrm{g}, \mathrm{h} \in \mathrm{G}, \mathrm{x} \in \mathscr{M} \text {. }
$$

(Such a mapping $\tau$ is called a representation of $\mathrm{G}$ in $\mathscr{M}$.)

(ii) $\mathscr{M}$ is called a G-manifold (of class $C^{k}$ ) if and only if for each $g \in G$ the mapping $\tau_{\mathrm{g}}: \mathscr{M} \rightarrow \mathscr{M}$ is a $\mathrm{C}^{\mathrm{k}}$-diffeomorphism. If $\mathrm{G}$ is an infinite Lie group then it is additionally required that the representation $\tau: G \times \mathscr{M} \rightarrow \mathscr{M}$ is of class $C^{k}$ for $\mathscr{M}$ to be a G-manifold.

(iii) For a G-manifold the subset of G-symmetric points, or in short the G-symmetric subset $\Sigma \subset \mathscr{M}$ is defined as

$$
\Sigma:=\left\{x \in \mathscr{M}: \tau_{\mathrm{g}}(\mathrm{x})=\mathrm{x} \text { for all } \mathrm{g} \in \mathrm{G}\right\} .
$$

(iv) A function $E: \mathscr{M} \rightarrow \mathbb{R}$ is G-invariant if and only if

$$
\mathrm{E}\left(\tau_{g}(x)\right)=\mathrm{E}(x) \text { for all } g \in \mathrm{G}, x \in \mathscr{M} \text {. }
$$

Now, Palais' principle of symmetric criticality reads as follows; cf. [27, Thm.5.4].

Theorem 2.4 (Palais). Let $\mathrm{G}$ be a compact Lie group and $\mathscr{M}$ a G-manifold of class $\mathrm{C}^{1}$ over the Banach space $\mathscr{B}$ with $\mathrm{G}$-symmetric subset $\Sigma \subset \mathscr{M}$, and let $\mathrm{E}: \mathscr{M} \rightarrow \mathbb{R}$ be a $\mathrm{G}$-invariant function of class $\mathrm{C}^{1}$. Then $\Sigma$ is a $\mathrm{C}^{1}$-submanifold of $\mathscr{M}$, and $x \in \Sigma$ is a critical point of $\mathrm{E}$ if and only if $x$ is critical for $\left.\mathrm{E}\right|_{\Sigma}: \Sigma \rightarrow \mathbb{R}$.

Since any finite group is a Lie group [11, p. 48, Example 5] one immediately obtains the following result which will be of relevance in our application.

Corollary 2.5. If $\mathrm{G}$ is a finite group, $\mathscr{M}$ a G-manifold of class $\mathrm{C}^{1}$ over the Banach space $\mathscr{B}$ with $\mathrm{G}$-symmetric subset $\Sigma \subset \mathscr{M}$, and if $\mathrm{E}: \mathscr{M} \rightarrow \mathbb{R}$ is a $\mathrm{G}$-invariant function of class $\mathrm{C}^{1}$, then $\mathrm{x} \in \Sigma$ is $\mathrm{E}$-critical if and only if it is $\left.\mathrm{E}\right|_{\Sigma}$-critical.

Remark 2.6. In our application the Banach manifold $\mathscr{M}$ will be an open subset $\Omega \subset \mathscr{B}$ of a Banach space $\mathscr{B}$, so that the differential of a $C^{1}$-function $E: \Omega \rightarrow \mathbb{R}$ coincides with the classic Fréchet-differential

$$
\mathrm{dE}_{\mathrm{x}}: \mathrm{T}_{\mathrm{x}} \Omega \simeq \mathscr{B} \rightarrow \mathrm{T}_{\mathrm{E}(\mathrm{x})} \mathbb{R} \simeq \mathbb{R},
$$

which may be calculated using the first variation, or Gâteaux-derivative:

$$
\mathrm{dE}_{x}[\mathrm{~h}]=\delta \mathrm{E}(\mathrm{x}, \mathrm{h}):=\lim _{\varepsilon \rightarrow 0} \frac{\mathrm{E}(\mathrm{x}+\varepsilon \mathrm{h})-\mathrm{E}(\mathrm{x})}{\varepsilon} \text { for } \mathrm{h} \in \mathscr{B} .
$$

Theorem 2.4 then implies that in order to establish criticality of a point $x \in \Sigma$ it suffices to show

$$
\mathrm{dE}_{x}[\mathrm{~h}]=0 \text { for all } h \in \mathrm{T}_{x} \Sigma \text {, }
$$

and not for all $h \in \mathscr{B}$.

\section{Properties of O’Hara's knot energies $E_{\alpha}$}

We start with the following bi-Lipschitz estimate due to O'Hara [25, Theorem 2.3], whose proof we present here for the convenience of the reader. 
Lemma 3.1. Any $\gamma \in C^{0,1}\left(\mathbb{R} / \mathbb{Z}, \mathbb{R}^{3}\right)$ with $\left|\gamma^{\prime}\right|>0$ a.e. and with $\mathrm{E}_{\alpha}(\gamma)<\infty$ for some $\alpha \in[2,3)$ is injective. More precisely, for all $\mathrm{b} \geqslant 0$ there is a constant $\mathrm{C}=\mathrm{C}(\mathrm{b}) \geqslant 0$ such that $\mathrm{E}_{\alpha}(\gamma) \leqslant \mathrm{b}$ implies the bi-Lipschitz estimate

$$
|\gamma(\mathrm{s})-\gamma(\mathrm{t})| \geqslant \mathrm{Cd}_{\gamma}(\mathrm{s}, \mathrm{t}) \text { for all } \mathrm{s}, \mathrm{t} \in \mathbb{R} / \mathbb{Z}
$$

Proof. Since $\left|\gamma^{\prime}\right|>0$ a.e. there is a one-to-one correspondence between the original parameters $s, t \in \mathbb{R} / \mathbb{Z}$ and the respective arclength parameters $\sigma(s)=\int_{0}^{s}\left|\gamma^{\prime}(\tau)\right| d \tau$ and $\sigma(t)=\int_{0}^{t}\left|\gamma^{\prime}(\tau)\right| d \tau$, so we may assume without loss of generality that $\gamma$ is already parametrized according to arclength, i.e., $\left|\gamma^{\prime}(\tau)\right|=1$ for a.e. $\tau \in \mathbb{R} / \mathbb{Z}$, and (by a parameter shift) that

$$
0 \leqslant s<t \leqslant s+\frac{1}{2} \text {. }
$$

Consequently, $(t-s)=|s-t|$ which equals the intrinsic distance

$$
\mathrm{d}_{\gamma}(\mathrm{s}, \mathrm{t})=|\mathrm{s}-\mathrm{t}|_{\mathbb{R} / \mathbb{Z}}:=\min \{|s-\mathrm{t}|, 1-|s-\mathrm{t}|\} .
$$

Setting

$$
\mathrm{d}:=|\gamma(\mathrm{s})-\gamma(\mathrm{t})| \text { and } \delta:=(\mathrm{t}-\mathrm{s})
$$

we assume first that $d \leqslant \delta / 4$, so that we can estimate for $0 \leqslant u, v \leqslant \delta / 8$

$$
|\gamma(s+u)-\gamma(t-v)| \leqslant d+u+v
$$

and

$$
|(t-v)-(s+u)|=(t-s)-(u+v)=\delta-(u+v) \geqslant \frac{3}{4} \delta
$$

where, again, the left-hand side equals the intrinsic distance $d_{\gamma}(s+u, t-v)$. By means of (5) and (6) we may now bound the energy from below to obtain

$$
\begin{aligned}
& b \geqslant \int_{s}^{s+\frac{\delta}{8}} \int_{0}^{t-\frac{\delta}{8}}\left(\frac{1}{|\gamma(x)-\gamma(y)|^{\alpha}}-\frac{1}{d_{\gamma}(x, y)^{\alpha}}\right) d y d x \\
& =\int_{0}^{\frac{\delta}{8}} \int_{0}^{\frac{\delta}{8}}\left(\frac{1}{|\gamma(s+u)-\gamma(t-v)|^{\alpha}}-\frac{1}{|(t-v)-(s+u)|^{\alpha}}\right) d u d v \\
& \stackrel{\text { [5, (6) }}{\geqslant} \int_{0}^{\frac{\delta}{8}} \int_{0}^{\frac{\delta}{8}}\left(\frac{1}{(d+u+v)^{\alpha}}-\frac{1}{\left(\frac{3}{4} \delta\right)^{\alpha}}\right) d u d v \\
& =\int_{0}^{\frac{\delta}{8}} \int_{0}^{\frac{\delta}{8}} \frac{1}{(d+u+v)^{\alpha}}\left[1-\left(\frac{d+u+v}{\frac{3}{4} \delta}\right)^{\alpha}\right] d u v .
\end{aligned}
$$

To estimate the term in square brackets in (7) notice that $d+u+v \leqslant d+(\delta / 4) \leqslant \delta / 2$ so that $(d+u+v) /(3 \delta / 4) \leqslant 2 / 3$, from which we infer

$$
\mathrm{b} \geqslant\left(1-\left(\frac{2}{3}\right)^{\alpha}\right) \int_{0}^{\frac{\delta}{8}} \int_{0}^{\frac{\delta}{8}} \frac{1}{(\mathrm{~d}+\mathfrak{u}+v)^{2}} \mathrm{dud} v=\left(1-\left(\frac{2}{3}\right)^{\alpha}\right) \log \frac{\left(\mathrm{d}+\frac{\delta}{8}\right)^{2}}{\mathrm{~d}\left(\mathrm{~d}+\frac{\delta}{4}\right)}
$$

by explicit integration. With $d+(\delta / 8) \geqslant(d+(\delta / 4)) / 2$ we can bound the argument of the logarithm by $\delta /(16 \mathrm{~d})$ from below to obtain

$$
b \geqslant\left(1-\left(\frac{2}{3}\right)^{\alpha}\right) \log \frac{\delta}{16 \mathrm{~d}},
$$


which leads to $e^{\mathrm{b} /\left(1-(2 / 3)^{\alpha}\right)} \geqslant \delta /(16 \mathrm{~d})$ or

$$
d \geqslant \frac{1}{16} e^{-b /\left(1-(2 / 3)^{\alpha}\right)} \delta \quad \text { if } d \leqslant \delta / 4 .
$$

This verifies our claim with constant

$$
\mathrm{C}:=\min \left\{\frac{1}{4}, \frac{1}{16} e^{-\mathrm{b} /\left(1-(2 / 3)^{\alpha}\right)}\right\}=\frac{1}{16} e^{-\mathrm{b} /\left(1-(2 / 3)^{\alpha}\right)} .
$$

Crucial for the application of Palais' principle of symmetric criticality is the identification of a suitable Banach manifold in our context of knotted curves and O'Hara's energy $E_{\alpha}$. This will be an open subset of an appropriate Sobolev-Slobodetckij space, which according to the important contribution of Blatt [4] - characterizes curves of finite $E_{\alpha^{-}}$ energy. Here is a slightly refined statement of Blatt's theorem.

Theorem 3.2 (Blatt). For any $\alpha \in[2,3)$ the following is true.

(i) If $\gamma \in \mathrm{C}^{0,1}\left(\mathbb{R} / \mathbb{Z}, \mathbb{R}^{3}\right)$ with length $0<\mathrm{L}:=\mathscr{L}(\gamma)$ satisfies $\left|\gamma^{\prime}\right|>0$ a.e. and $\mathrm{E}_{\alpha}(\gamma)<$ $\infty$, then $\left.\gamma\right|_{[0,1)}$ is injective, and its arclength parametrization $\Gamma \in \mathrm{C}^{0,1}\left(\mathbb{R} /(\mathrm{L}), \mathbb{R}^{3}\right)$ is of class $W^{(\alpha+1) / 2,2}\left(\mathbb{R} /(\mathrm{L} \mathbb{Z}), \mathbb{R}^{3}\right)$ with unit tangent $\Gamma^{\prime}$ satisfying

$$
\left[\Gamma^{\prime}\right]_{(\alpha-1) / 2,2}^{2} \leqslant 4^{4} \cdot 2^{2-2 \alpha} \mathrm{E}_{\alpha}(\gamma) .
$$

(ii) If, on the other hand, $\alpha \in(2,3)$ and $\gamma \in W^{(\alpha+1) / 2,2}\left(\mathbb{R} / \mathbb{Z}, \mathbb{R}^{3}\right)$ with $\left|\gamma^{\prime}\right|>0$ a.e., and if $\left.\gamma\right|_{[0,1)}$ is injective, then $\mathrm{E}_{\alpha}(\gamma)<\infty$.

Blatt actually proved part (ii) only for arclength parametrized curves, but for the full two-parameter family of O'Hara's energies which also includes the case $\alpha=2$.

Before giving the proof of Theorem 3.2 let us quickly recall the concept of SobolevSlobodetckij spaces, where it suffices for our applications to focus on the case of periodic functions of one variable. For that we define for fixed $L>0, s \in(0,1)$ and $\rho \in[1, \infty)$ the seminorm

$$
[\mathrm{f}]_{s, \rho}:=\left(\int_{\mathbb{R} /(\mathrm{LZ})} \int_{-\mathrm{L} / 2}^{\mathrm{L} / 2} \frac{|\mathrm{f}(\mathrm{u}+w)-\mathrm{f}(\mathrm{u})|^{\rho}}{|w|^{1+\rho s}} \mathrm{~d} w \mathrm{du}\right)^{1 / \rho}
$$

for an integrable function $f \in L^{\rho}\left(\mathbb{R} /(L \mathbb{Z}), \mathbb{R}^{n}\right)$, which explains our notation in (9)).

Definition 3.3. For $k \in \mathbb{N}$, the set

$$
W^{k+s, \rho}\left(\mathbb{R} /(L \mathbb{Z}), \mathbb{R}^{n}\right):=\left\{f \in W^{k, \rho}\left(\mathbb{R} /(L \mathbb{Z}), \mathbb{R}^{n}\right):\|f\|_{W^{k+s, \rho}}<\infty\right\},
$$

where

$$
\|f\|_{W^{k+s, \rho}}:=\|f\|_{W^{k, \rho}}+\left[f^{(k)}\right]_{s, \rho},
$$

is called the Sobolev-Slobodetckij space with (fractional) differentiability order $k+s$ and integrability $\rho$. (Here, $W^{k, \rho}$ denotes the usual Sobolev space of functions whose generalized derivatives up to order $k$ are $\rho$-integrable.) 
Remark 3.4. It is well-known that Sobolev-Slobodetckij are Banach spaces, and one has the following continuous Morrey-type embedding 1 into classical Hölder spaces:

$$
W^{k+s, \rho}\left(\mathbb{R} /(\mathrm{LZ}), \mathbb{R}^{n}\right) \hookrightarrow C^{k, s-(1 / \rho)}\left(\mathbb{R} /(\mathrm{L} \mathbb{Z}), \mathbb{R}^{n}\right) \text { for } \rho \in(1, \infty), s \in(1 / \rho, 1) .
$$

In our context we obtain for $\alpha \in(2,3), s=(\alpha-1) / 2 \in(1 / 2,1)$, and $\rho=2$ the continuous embedding

$$
\begin{aligned}
W^{(\alpha+1) / 2,2}\left(\mathbb{R} /(\mathrm{L} Z), \mathbb{R}^{\mathrm{n}}\right)=\mathrm{W}^{1+s, 2}\left(\mathbb{R} /(\mathrm{L} \mathbb{Z}), \mathbb{R}^{\mathrm{n}}\right) & \hookrightarrow \mathrm{C}^{1, \mathrm{~s}-(1 / 2)}\left(\mathbb{R} /(\mathrm{L} \mathbb{Z}), \mathbb{R}^{\mathrm{n}}\right) \\
& =\mathrm{C}^{1,(\alpha / 2)-1}\left(\mathbb{R} /(\mathrm{L} \mathbb{Z}), \mathbb{R}^{\mathrm{n}}\right),
\end{aligned}
$$

which means that there is a constant $C_{E}=C_{E}(L, n)$ such that

$$
\|f\|_{C^{1,(\alpha / 2)-1}} \leqslant C_{E}\|f\|_{W^{(\alpha+1) / 2,2}} \text { for all } f \in W^{(\alpha+1) / 2,2}\left(\mathbb{R} /(L \mathbb{Z}), \mathbb{R}^{n}\right) .
$$

This uniform estimate will turn out to be quite useful in our context, e.g., to obtain compactness, or to conserve the prescribed knot class in the limit of minimal sequences; see Section 4, in particular the proof of Theorem 4.9.

Proof of Theorem 3.2 (i) Injectivity follows from Lemma 3.1. Since $\mathrm{E}_{\alpha}$ is invariant under reparametrization we have $\mathrm{E}_{\alpha}(\gamma)=\mathrm{E}_{\alpha}(\Gamma)$. So, we can estimate

$$
\begin{aligned}
& \infty>\mathrm{E}_{\alpha}(\Gamma)=\int_{\mathbb{R} /(\mathrm{LZ})} \int_{-\mathrm{L} / 2}^{\mathrm{L} / 2}\left(\frac{1-\frac{|\Gamma(\mathrm{u}+w)-\Gamma(\mathrm{u})|^{\alpha}}{\mid w^{\mid}}}{|w|^{\alpha}}\right) \frac{|w|^{\alpha}}{|\Gamma(\mathrm{u}+w)-\Gamma(\mathrm{u})|^{\alpha}} \mathrm{d} w \mathrm{~d} u \\
& \geqslant \int_{\mathbb{R} /(\mathrm{LZ})} \int_{-\mathrm{L} / 2}^{\mathrm{L} / 2} \frac{1-\frac{|\Gamma(\mathrm{u}+w)-\Gamma(\mathfrak{u})|^{2}}{|w|^{2}}}{|w|^{\alpha}} \mathrm{d} w \mathrm{~d} \mathrm{u},
\end{aligned}
$$

where we have used that the arclength parametrization $\Gamma$ is Lipschitz continuous with Lipschitz constant 1 , and $\alpha \geqslant 2$. Now, the numerator of the last integral may be rewritten as

(14) $\int_{0}^{1} \int_{0}^{1}\left(1-\Gamma^{\prime}(u+\sigma w) \cdot \Gamma^{\prime}(u+\tau w)\right) d \sigma d \tau=\frac{1}{2} \int_{0}^{1} \int_{0}^{1}\left|\Gamma^{\prime}(u+\sigma w)-\Gamma^{\prime}(u+\tau w)\right|^{2} d \sigma d \tau$,

which - inserted into (13) and combined with Fubini's theorem - leads to the following lower bound for $\mathrm{E}_{\alpha}(\Gamma)$ :

$$
\frac{1}{2} \int_{0}^{1} \int_{0}^{1} \int_{-\mathrm{L} / 2}^{\mathrm{L} / 2} \int_{0}^{\mathrm{L}} \frac{\left|\Gamma^{\prime}(u+\sigma w)-\Gamma^{\prime}(u+\tau w)\right|^{2}}{|w|^{\alpha}} \mathrm{dud} w \mathrm{~d} \sigma \mathrm{d} \tau
$$

which can be transformed via the substitution $z:=u+\sigma w$ into

$$
\frac{1}{2} \int_{0}^{1} \int_{0}^{1} \int_{-\mathrm{L} / 2}^{\mathrm{L} / 2} \int_{\sigma w}^{\mathrm{L}+\sigma w} \frac{\left|\Gamma^{\prime}(z)-\Gamma^{\prime}(z+(\tau-\sigma) w)\right|^{2}}{|w|^{\alpha}} \mathrm{d} z \mathrm{~d} w \mathrm{~d} \sigma \mathrm{d} \tau
$$

By L-periodicity we may replace the inner integration by the integral on $\mathbb{R} /(\mathrm{L} \mathbb{Z})$, and we estimate the resulting quadruple integral from below by restricting the integration with respect to $\tau$ to the interval $[3 / 4,1]$ and the $\sigma$-integration to $[0,1 / 4]$, before we interchange

\footnotetext{
${ }^{1}$ For this and many more advanced facts on fractional Sobolev spaces we refer, e.g., to [29], [13], [2], or to the monographs [35 37]
} 
the inner two integrations with Fubini and substitute then $y:=(\tau-\sigma) \mathcal{w}$, to arrive at the new lower bound for $E_{\alpha}(\Gamma)$ :

$$
\frac{1}{2} \int_{3 / 4}^{1} \int_{0}^{1 / 4}(\tau-\sigma)^{\alpha-1} \int_{\mathbb{R} /(\mathrm{LZ})} \int_{-(\tau-\sigma) \mathrm{L} / 2}^{(\tau-\sigma) \mathrm{L} / 2} \frac{\left|\Gamma^{\prime}(z)-\Gamma^{\prime}(z+y)\right|^{2}}{|y|^{\alpha}} \mathrm{d} y \mathrm{~d} z \mathrm{~d} \sigma \mathrm{d} \tau,
$$

which itself is bounded from below by

$$
\frac{1}{2}\left(\frac{1}{4}\right)^{3} \int_{\mathbb{R} /(\mathrm{LZ})} \int_{-\mathrm{L} / 4}^{\mathrm{L} / 4} \frac{\left|\Gamma^{\prime}(z)-\Gamma^{\prime}(z+y)\right|^{2}}{|y|^{\alpha}} \mathrm{d} y \mathrm{~d} z .
$$

The Sobolev-Slobodetckij seminorm (10) for $s=(\alpha-1) / 2$ and therefore $1+2 s=\alpha$, on the other hand, may be estimated by means of the triangle inequality as

$$
\begin{aligned}
{\left[\Gamma^{\prime}\right]_{(\alpha-1) / 2,2}^{2}=} & \int_{\mathbb{R} /(\mathrm{LZ})} \int_{-\mathrm{L} / 2}^{\mathrm{L} / 2} \frac{\left|\Gamma^{\prime}(z+x)-\Gamma^{\prime}(z)\right|^{2}}{|x|^{\alpha}} \mathrm{d} x \mathrm{~d} z \\
\leqslant & 2 \int_{\mathbb{R} /(\mathrm{LZ})} \int_{-\mathrm{L} / 2}^{\mathrm{L} / 2} \frac{\left|\Gamma^{\prime}(z+x)-\Gamma^{\prime}(z+(x / 2))\right|^{2}}{|x|^{\alpha}} \mathrm{d} x \mathrm{~d} z \\
& +2 \int_{\mathbb{R} /(\mathrm{L} \mathbb{Z})} \int_{-\mathrm{L} / 2}^{\mathrm{L} / 2} \frac{\left|\Gamma^{\prime}(z+(\mathrm{x} / 2))-\Gamma^{\prime}(z)\right|^{2}}{|x|^{\alpha}} \mathrm{d} x \mathrm{~d} z .
\end{aligned}
$$

Substituting $y:=x / 2$ transforms the second double integral on the right-hand side into

$$
2^{1-\alpha} \int_{\mathbb{R} /(\mathrm{LZ})} \int_{-\mathrm{L} / 4}^{\mathrm{L} / 4} \frac{\left|\Gamma^{\prime}(z+y)-\Gamma^{\prime}(z)\right|^{2}}{|y|^{\alpha}} \mathrm{dy} \mathrm{d} z .
$$

In the first integral on the right-hand side of (18) we first use Fubini to interchange the order of integration, then the substitution $\zeta:=z+x$ in the $z$-integral to arrive at

$$
\int_{-\mathrm{L} / 2}^{\mathrm{L} / 2} \int_{x}^{\mathrm{L}+\mathrm{x}} \frac{\left|\Gamma^{\prime}(\zeta)-\Gamma^{\prime}(\zeta-(x / 2))\right|^{2}}{|x|^{\alpha}} \mathrm{d} \zeta \mathrm{d} x=\int_{-\mathrm{L} / 2}^{\mathrm{L} / 2} \int_{\mathbb{R} /(\mathrm{LZ})} \frac{\left|\Gamma^{\prime}(\zeta)-\Gamma^{\prime}(\zeta-(x / 2))\right|^{2}}{|x|^{\alpha}} \mathrm{d} \zeta \mathrm{d} x,
$$

where we used L-periodicity of $\Gamma^{\prime}$. Interchanging the order of integration again, and then substituting here $y:=-x / 2$ in the $x$-integration finally leads to the term (19) again. Thus, inserting (19) for both double integrals on the right-hand side of (18), and combining this with (17) we obtain the desired energy estimate (9).

(ii) By Lemma A.1 also the arclength parametrization $\Gamma: \mathbb{R} /(\mathrm{LZ}) \rightarrow \mathbb{R}^{3}$ of $\gamma$ is of class $W^{(\alpha+1) / 2,2}$ with the estimate (56), where $L=\mathscr{L}(\gamma)$ denotes the length of $\gamma$. So, it suffices to work with $\Gamma$ due to the parameter invariance of $E_{\alpha}$. In addition, we prove in the appendix (see Corollary A.3) that $\Gamma$ is bi-Lipschitz continuous satisfying

$$
\frac{1}{\mathrm{~B}}|w| \leqslant|\Gamma(u+w)-\Gamma(u)| \leqslant|w| \text { for all } u \in \mathbb{R} /(\mathrm{LZ}),|w| \leqslant \mathrm{L} / 2
$$

for some constant $B=B(\alpha, \Gamma)$ depending on $\alpha$ and on the curve $\Gamma$. Similarly as in the proof of part (i) we first rewrite the energy of $\Gamma$ as

$$
\begin{aligned}
& \mathrm{E}_{\alpha}(\Gamma)=\int_{\mathbb{R} /(\mathrm{LZ})} \int_{-\mathrm{L} / 2}^{\mathrm{L} / 2}\left(\frac{1-\frac{|\Gamma(\mathfrak{u}+w)-\Gamma(\mathfrak{u})|^{\alpha}}{\mid w^{\alpha}}}{|w|^{\alpha}}\right) \frac{|w|^{\alpha}}{|\Gamma(\mathrm{u}+w)-\Gamma(\mathfrak{u})|^{\alpha}} \mathrm{d} w \mathrm{du} \\
& \leqslant \mathrm{B}^{\alpha} \int_{\mathbb{R} /(\mathrm{L} \mathbb{Z})} \int_{-\mathrm{L} / 2}^{\mathrm{L} / 2} \frac{1-\frac{|\Gamma(\mathfrak{u}+w)-\Gamma(\mathfrak{u})|^{\alpha}}{|w|^{\alpha}}}{|w|^{\alpha}} \mathrm{d} w \mathrm{du},
\end{aligned}
$$


where we used (20) for the inequality. By the elementary inequality

$$
1-\chi^{\alpha} \leqslant(\alpha+1)\left(1-\chi^{2}\right) \text { for all } \alpha \in[2, \infty), x \in[0,1]
$$

proved in Lemma A.4 in the appendix we can estimate the right-hand side of (21) from above by

$$
(\alpha+1) B^{\alpha} \int_{\mathbb{R} /(\mathrm{LZ})} \int_{-\mathrm{L} / 2}^{\mathrm{L} / 2} \frac{1-\frac{|\Gamma(\mathrm{u}+w)-\Gamma(u)|^{2}}{|w|^{2}}}{|w|^{\alpha}} \mathrm{d} w \mathrm{du} .
$$

This double integral is identical with the one in (13), so we can perform exactly the same manipulations using Fubini and one substitution as in (14), (15), (16), to rewrite (22) as

$$
\frac{1}{2}(\alpha+1) \mathrm{B}^{\alpha} \int_{0}^{1} \int_{0}^{1} \int_{-\mathrm{L} / 2}^{\mathrm{L} / 2} \int_{\sigma w}^{\mathrm{L}+\sigma w} \frac{\left|\Gamma^{\prime}(z)-\Gamma^{\prime}(z+(\tau-\sigma) w)\right|^{2}}{|w|^{\alpha}} \mathrm{d} z \mathrm{~d} w \mathrm{~d} \sigma \mathrm{d} \tau
$$

where in the $z$-integration we may replace the domain of integration by $\mathbb{R} /(\mathrm{L} \mathbb{Z})$ due to L-periodicity of $\Gamma$. Exchanging the order of the $z$-integration with the $w$-integration we can substitute $y(w):=(\tau-\sigma) w$ to obtain

$$
\frac{1}{2}(\alpha+1) \mathrm{B}^{\alpha} \int_{0}^{1} \int_{0}^{1}|\tau-\sigma|^{\alpha-1} \int_{\mathbb{R} /(\mathrm{LZ})} \int_{-|\tau-\sigma| \mathrm{L} / 2}^{|\tau-\sigma| \mathrm{L} / 2} \frac{\left|\Gamma^{\prime}(z)-\Gamma^{\prime}(z+y)\right|^{2}}{|y|^{\alpha}} \mathrm{d} y \mathrm{~d} z \mathrm{~d} \sigma \mathrm{d} \tau
$$

where the integration domain of the $y$-integration may be replaced by the full interval $[-\mathrm{L} / 2, \mathrm{~L} / 2]$ since $|\tau-\sigma| \leqslant 1$, giving

$$
\frac{1}{2}(\alpha+1) \mathrm{B}^{\alpha} \int_{0}^{1} \int_{0}^{1} \int_{\mathbb{R} /(\mathrm{LZ})} \int_{-\mathrm{L} / 2}^{\mathrm{L} / 2} \frac{\left|\Gamma^{\prime}(z)-\Gamma^{\prime}(z+y)\right|^{2}}{|y|^{\alpha}} \mathrm{d} y \mathrm{~d} z \mathrm{~d} \sigma \mathrm{d} \tau=\frac{1}{2}(\alpha+1) \mathrm{B}^{\alpha}\left[\Gamma^{\prime}\right]_{(\alpha-1) / 2,2}^{2}
$$

as an upper bound for $E_{\alpha}(\Gamma)$. Combining this with (56) in Lemma A.1 in the appendix we conclude

$$
\begin{aligned}
\mathrm{E}_{\alpha}(\gamma)=\mathrm{E}_{\alpha}(\Gamma) & \leqslant \frac{1}{2}(\alpha+1) \mathrm{B}^{\alpha}\left[\Gamma^{\prime}\right]_{(\alpha-1) / 2,2}^{2} \\
& \stackrel{(56)}{\leqslant} \frac{1}{2}(\alpha+1) \mathrm{B}^{\alpha}\left(\frac{1}{\mathrm{c}}\right)^{2+\alpha}\left[\left(\frac{1}{\mathrm{c}}\right)^{2}+\mathrm{C}^{6}\right] \cdot\left[\gamma^{\prime}\right]_{(\alpha-1) / 2,2}^{2},
\end{aligned}
$$

where $c=\min _{[0,1]}\left|\gamma^{\prime}\right|$ and $C=\max _{[0,1]}\left|\gamma^{\prime}\right|$, which finishes the proof.

Lower semicontinuity of $E_{\alpha}$ was shown in the case $\alpha=2$ by Freedman, He, and Wang in [15, Lemma 4.2], and their argument works also for any $\alpha \in[2,3)$.

Lemma 3.5. Let $\alpha \in[2,3)$ and assume that $\gamma, \gamma_{i}: \mathbb{R} / \mathbb{Z} \rightarrow \mathbb{R}^{n}$ are absolutely continuous curves with $\left|\gamma^{\prime}\right|>0$ and $\left|\gamma_{i}^{\prime}\right|>0$ a.e. on $\mathbb{R} / \mathbb{Z}$ for all $i \in \mathbb{N}$, such that $\gamma_{i} \rightarrow \gamma$ pointwise everywhere on $\mathbb{R} / \mathbb{Z}$ as $i \rightarrow \infty$. Then

$$
E_{\alpha}(\gamma) \leqslant \liminf _{i \rightarrow \infty} E_{\alpha}\left(\gamma_{i}\right) .
$$

Proof. We may assume that the liminf on the right-hand side is finite, and that it is realized as the limit $E_{\alpha}\left(\gamma_{i}\right)$ (upon restriction to a subsequence again denoted by $\gamma_{i}$ ). It is well-known that the length functional $\mathscr{L}$ is lower semicontinuous with respect to pointwise convergence, so that also $d_{\gamma}(u+w, u) \leqslant \liminf _{i \rightarrow \infty} d_{\gamma_{i}}(u+w, u)$; hence

$$
\limsup _{i \rightarrow \infty} \frac{1}{d_{\gamma_{i}}(u+w, u)} \leqslant \frac{1}{d_{\gamma}(u+w, u)} \text { for all } u \in \mathbb{R} / \mathbb{Z},|w| \leqslant 1 / 2 \text {. }
$$


Together with the pointwise convergence $\left|\gamma_{i}(u+w)-\gamma_{\mathfrak{i}}(u)\right| \rightarrow|\gamma(u+w)-\gamma(u)|$ as $i \rightarrow \infty$ we obtain

$$
\begin{aligned}
& \frac{1}{|\gamma(u+w)-\gamma(u)|^{\alpha}}-\frac{1}{d_{\gamma}(u+w, u)^{\alpha}} \leqslant \\
& \liminf _{i \rightarrow \infty}\left(\frac{1}{\left|\gamma_{i}(u+w)-\gamma_{i}(u)\right|^{\alpha}}-\frac{1}{d_{\gamma_{i}}(u+w, u)^{\alpha}}\right) \text { for all } u \in \mathbb{R} / \mathbb{Z},|w| \leqslant 1 / 2 .
\end{aligned}
$$

In addition, using again the lower semicontinuity of length, we can estimate for any $0<$ $h \ll 1$ and any $s \in \mathbb{R} / \mathbb{Z}$,

$$
|\gamma(s+h)-\gamma(s)| \leqslant d_{\gamma}(s+h, s) \leqslant \liminf _{i \rightarrow \infty} d_{\gamma_{i}}(s+h, s)=\liminf _{i \rightarrow \infty} \int_{s}^{s+h}\left|\gamma_{i}^{\prime}(\tau)\right| d \tau .
$$

Dividing this inequality by $h$ and taking the limit $h \searrow 0$ we obtain at differentiability points $s$ of $\gamma$ that are also Lebesgue points of all $\left|\gamma_{i}^{\prime}\right|$ simultaneously - hence for a.e. $s \in \mathbb{R} / \mathbb{Z}$ - the limiting inequality

$$
\left|\gamma^{\prime}(s)\right| \leqslant \liminf _{i \rightarrow \infty}\left|\gamma_{i}^{\prime}(s)\right| .
$$

Combining (26) with (27) we obtain that the integrand of $E_{\alpha}$ is bounded from above by the limes inferior of the integrands of $E_{\alpha}\left(\gamma_{i}\right)$ as $i \rightarrow \infty$. This together with Fatou's Lemma and the monotonicity of the integral proves the claim.

Remark 3.6. In [7, Theorem 1.1] Blatt and Reiter prove that $E_{\alpha}$ is continuously differentiable on the space of all injective regular curves of class $W^{(\alpha+1) / 2,2}$, and they give an explicit formula of the differential $\mathrm{dE}_{\gamma}[\cdot]$ in the case of an arclength parametrized curve $\gamma \in W^{(\alpha+1) / 2,2}\left(\mathbb{R} / \mathbb{Z}, \mathbb{R}^{n}\right)$. The explicit structure of this differential is not needed in our context, but the differentiability of $E$ is, of course, crucial to apply Palais' principle of symmetric criticality to obtain classic critical points - in contrast, e.g. to the notion of criticality for the non-smooth ropelength functional formulated by Cantarella et al. in [10]. Moreover, Blatt and Reiter's main theorem [7, Theorem 1.2] states that any arclength parametrized critical point of the linear combination $\mathrm{E}_{\alpha}+\lambda \mathscr{L}$ is $\mathrm{C}^{\infty}$-smooth. Here, $\mathscr{L}$ denotes as before the length functional, and $\lambda \in \mathbb{R}$ is an arbitrary parameter, that, e.g., comes up as a Lagrange parameter for a minimization problem for $E_{\alpha}$ under a fixed length constraint. Alternatively, and important for our construction of symmetric critical points in Section 4, such a scalar parameter appears if one considers the scale-invariant version $S_{\alpha}$ of $E_{\alpha}$ defined in (2) in the introduction. The differential of $S_{\alpha}$ evaluated at some injective regular curve $\gamma \in W^{(\alpha+1) / 2,2}\left(\mathbb{R} / \mathbb{Z}, \mathbb{R}^{n}\right)$ has the form

$$
\mathrm{d}\left(\mathrm{S}_{\alpha}\right)_{\gamma}=\mathrm{d}\left(\mathscr{L}^{\alpha-2} \mathrm{E}_{\alpha}\right)_{\gamma}=\mathscr{L}(\gamma)^{\alpha-2} \mathrm{~d}\left(\mathrm{E}_{\alpha}\right)_{\gamma}+\left((\alpha-2) \mathscr{L}(\gamma)^{\alpha-1} \mathrm{E}_{\alpha}(\gamma)\right) \mathrm{d} \mathscr{L}_{\gamma} .
$$

Hence Blatt and Reiter's regularity theorem applies to any arclength parametrized critical point $\gamma$ of $S_{\alpha}$ (setting $\lambda:=(\alpha-2) \mathscr{L}(\gamma) E_{\alpha}(\gamma)$ ) implying the smoothness of such $\gamma$.

\section{Critical torus knots}

We first establish an open subset of the Banach space $W^{(\alpha+1) / 2,2}\left(\mathbb{R} / \mathbb{Z}, \mathbb{R}^{3}\right)$ as the Banach manifold on which Palais' principle of symmetric criticality is applicable. 
Lemma 4.1. For any tame ${ }^{2}$ knot class $\mathcal{K}$ and for any $\alpha \in(2,3)$ the set

$$
\Omega_{\mathcal{K}}:=\left\{\gamma=\left(\gamma^{1}, \gamma^{2}, \gamma^{3}\right) \in W^{(\alpha+1) / 2,2}\left(\mathbb{R} / \mathbb{Z}, \mathbb{R}^{3}\right):\left|\gamma^{\prime}\right|>0,\left(\gamma^{1}\right)^{2}+\left(\gamma^{2}\right)^{2}>0,[\gamma]=\mathcal{K}\right\}
$$

is an open subset of $W^{(\alpha+1) / 2,2}\left(\mathbb{R} / \mathbb{Z}, \mathbb{R}^{3}\right)$.

(Here, $[\gamma]$ denotes the knot class represented by $\gamma$. In particular, $[\gamma]=\mathcal{K}$ implies automatically that $\left.\gamma\right|_{[0,1)}$ is injective.)

Corollary 4.2. The set $\Omega_{\mathcal{K}}$ defined in Lemma 4.1 is a smooth manifold modeled over the Banach space $\mathscr{B}:=W^{(\alpha+1) / 2,2}\left(\mathbb{R} / \mathbb{Z}, \mathbb{R}^{3}\right)$.

Proof of Lemma 4.1 Fix $\gamma \in \Omega_{\mathcal{K}}$, and notice that $\gamma$ is of class $C^{1,(\alpha / 2)-1}\left(\mathbb{R} / \mathbb{Z}, \mathbb{R}^{3}\right)$ since $\alpha>2$ so that the Morrey-type embedding holds; see (11). In particular, there is a constant $c_{\gamma}>0$ such that $\min \left\{\left|\gamma^{\prime}\right|, \sqrt{\left(\gamma^{1}\right)^{2}+\left(\gamma^{2}\right)^{2}}\right\} \geqslant c_{\gamma}$ on $[0,1]$. Thus, for every $h \in W^{(\alpha+1) / 2,2}\left(\mathbb{R} / \mathbb{Z}, \mathbb{R}^{3}\right)$ we find by means of (12)

$$
\begin{aligned}
\min \left\{\left|(\gamma+h)^{\prime}\right|, \sqrt{\left(\gamma^{1}+h^{1}\right)^{2}+\left(\gamma^{2}+h^{2}\right)^{2}}\right\} & \geqslant c_{\gamma}-\|h\|_{C^{1,(\alpha / 2)-1}} \\
& \stackrel{12)}{\geqslant} c_{\gamma}-C_{E}\|h\|_{W^{(\alpha+1) / 2,2}} \geqslant \frac{1}{2} c_{\gamma}>0,
\end{aligned}
$$

if $\|h\|_{W^{(\alpha+1) / 2,2}} \leqslant c_{\gamma} /\left(2 C_{E}\right)$, where $C_{E}=C_{E}(1,3)$ is the constant in the embedding inequality (12) in ambient space dimension $n=3$. According to the stability of the isotopy class under $C^{1}$-perturbations (see, e.g. [28] or [3]) there exists some $\varepsilon_{\gamma}>0$ such that all curves $\xi \in B_{\varepsilon_{\gamma}}(\gamma) \subset C^{1}\left(\mathbb{R} / \mathbb{Z}, \mathbb{R}^{3}\right)$ are ambient isotopic to $\gamma$. This implies that for any $h \in W^{(\alpha+1) / 2,2}\left(\mathbb{R} / \mathbb{Z}, \mathbb{R}^{3}\right)$ with $\|h\|_{W^{(\alpha+1) / 2,2}} \leqslant \varepsilon_{\gamma} / C_{E}$ we have $\gamma+h \in B_{\varepsilon_{\gamma}}(\gamma) \subset C^{1}\left(\mathbb{R} / \mathbb{Z}, \mathbb{R}^{3}\right)$, so that $[\gamma+h]=\mathcal{K}$. Setting $\delta:=\min \left\{\varepsilon_{\gamma}, c_{\gamma} / 2\right\} / C_{E}$ we conclude that the open ball $\mathrm{B}_{\delta}(\gamma) \subset \mathrm{W}^{(\alpha+1) / 2,2}\left(\mathbb{R} / \mathbb{Z}, \mathbb{R}^{3}\right)$ is actually contained in $\Omega_{\mathcal{K}}$.

Since we are going to look at symmetric knots under rotations with a fixed angle we are led to consider the finite cyclic group $\mathbb{Z} /(\mathrm{m} \mathbb{Z})$, for which we recall its definition.

Definition 4.3. For $m \in \mathbb{Z}$ with $|m| \geqslant 2$ let $G:=\mathbb{Z} /(m \mathbb{Z})$ be the subgroup of $(\mathbb{Z},+)$ consisting of the equivalence classes $[z]$ determined by the equivalence relation

$z_{1}, z_{2} \in \mathbb{Z}$ are equivalent denoted by $z_{1} \sim z_{2} \Longleftrightarrow z_{1}=z_{2}+\mathrm{km}$ for some $k \in \mathbb{Z}$.

The group $(G,+)$ forms a group with $m$ elements, where the addition is defined as $\left[z_{1}\right]+\left[z_{2}\right]=\left[z_{1}+z_{2}\right]$ which is well-defined since it does not depend on the choice of representatives.

As we deal with parametrized curves we need to adjust rotations in space by appropriate parameter shifts in the domain. To be precise we establish in the following lemma a set of group actions of $\mathrm{G}$ (depending on an additional integer parameter) on the Banach manifold $\Omega_{\mathcal{K}}$ for any given knot class $\mathcal{K}$. Here, and also later, we use the notation

$$
\operatorname{Rot}(\beta):=\left(\begin{array}{ccc}
\cos \beta & -\sin \beta & 0 \\
\sin \beta & \cos \beta & 0 \\
0 & 0 & 1
\end{array}\right) \in \operatorname{SO}(3)
$$

\footnotetext{
${ }^{2} \mathrm{~A}$ knot class is called tame if it contains polygonal loops. Any knot class containing $\mathrm{C}^{1}$-representatives is tame, see R. H. Crowell and R. H. Fox [12, App. I], and vice versa, any tame knot class contains smooth representatives.
} 
for the rotation matrix about the $z$-axis (with respect to the standard basis of $\mathbb{R}^{3}$ ), and we write, more generally, $\operatorname{Rot}(\beta, v)$ for a rotation about an arbitrary axis $v$ with rotational angle $\beta$. Notice that in that case $v$ does not necessarily contain the origin.

Lemma 4.4. Let $\mathcal{K}$ be an arbitrary tame knot class, and fix $\alpha \in(2,3), \mathrm{k}, \mathrm{m} \in \mathbb{Z}$, and let $\mathrm{G}:=\mathbb{Z} /(\mathrm{m} \mathbb{Z})$. Then $\mathrm{G}$ acts on $\Omega_{\mathcal{K}}$ via the mapping

$$
\begin{aligned}
\tau^{\mathrm{k}}: \mathrm{G} \times \Omega_{\mathcal{K}} & \longrightarrow \Omega_{\mathcal{K}} \\
(\mathrm{g}, \gamma) & \longmapsto \tau_{\mathrm{g}}^{\mathrm{k}}(\gamma)
\end{aligned}
$$

defined as

$$
\tau_{g}^{\mathrm{k}}(\gamma)(\mathrm{t}):=\mathrm{D}_{\mathrm{g}} \gamma\left(\mathrm{t}+\frac{\mathrm{k}}{\mathrm{m}} \cdot \mathrm{l}_{\mathrm{g}}\right) \text { for } \mathrm{t} \in \mathbb{R} / \mathbb{Z},
$$

where $\mathrm{D}_{\mathrm{g}}=\operatorname{Rot}\left(2 \pi \mathrm{l}_{\mathrm{g}} / \mathrm{m}\right) \in \mathrm{SO}(3)$ and $\mathrm{l}_{\mathrm{g}} \in \mathbb{Z}$ is a representative of $\mathrm{g} \in \mathrm{G}$. Moreover, $\Omega_{\mathcal{K}}$ becomes a smooth $\mathrm{G}$-manifold under this action.

Remark 4.5. As $\gamma$ is 1-periodic, $\tau_{g}^{k}$ in (29) is obviously well-defined since it does not depend on the choice of representative $l_{g}$, since any other representative differs from $l_{g}$ only by an integer multiple of $\mathrm{m}$.

Proof of Lemma 4.4. Since a rotation in the ambient space and a parameter shift does not change the Sobolev-Slobodetckij norm we find that $\tau_{\mathrm{g}}^{\mathrm{k}}(\gamma) \in W^{(\alpha+1) / 2,2}\left(\mathbb{R} / \mathbb{Z}, \mathbb{R}^{3}\right)$ for any $\gamma \in W^{(\alpha+1) / 2,2}\left(\mathbb{R} / \mathbb{Z}, \mathbb{R}^{3}\right)$. Moreover,

$$
\begin{aligned}
& \min \left\{\left|\tau_{g}^{k}(\gamma)^{\prime}(t)\right|, \sqrt{\left(\left(\tau_{g}^{k}(\gamma)\right)^{1}(t)\right)^{2}+\left(\left(\tau_{g}^{k}(\gamma)\right)^{2}(t)\right)^{2}}\right\} \\
& =\min \left\{\left|\gamma^{\prime}\left(t+\frac{k}{m} \cdot l_{g}\right)\right|, \sqrt{\left(\gamma^{1}\left(t+\frac{k}{m} \cdot l_{g}\right)\right)^{2}+\left(\gamma^{2}\left(t+\frac{k}{m} \cdot l_{g}\right)\right)^{2}}\right\}>0 \text { for all } t \in \mathbb{R} / \mathbb{Z} .
\end{aligned}
$$

A parameter shift combined with a rotation in ambient space does not change the knot type, that is, $\left[\tau_{\mathrm{g}}^{\mathrm{k}}(\gamma)\right]=\mathcal{K}$, so that $\tau_{\mathrm{g}}^{\mathrm{k}}(\gamma) \in \Omega_{\mathcal{K}}$ for any $\gamma \in \Omega_{\mathcal{K}}$. We need to check that $\tau^{\mathrm{k}}$ is a representation of $\mathrm{G}$ on $\Omega_{\mathcal{K}}$; cf. Definition 2.3. Indeed, for $\mathrm{g}, \mathrm{h} \in \mathrm{G}$ we may choose the representative $l_{g+h}=l_{g}+l_{h}$ as a representative for the group element $g+h \in G$, so that

$$
\begin{aligned}
\tau_{g+h}^{k}(\gamma)(t) & =D_{g+h} \gamma\left(t+\frac{k}{m} l_{g+h}\right)=D_{g} D_{h} \gamma\left(t+\frac{k}{m}\left(l_{g}+l_{h}\right)\right) \\
& =D_{g}\left(D_{h} \gamma\left(\cdot+\frac{k}{m} l_{h}\right)\right)\left(t+\frac{k}{m} l_{g}\right)=D_{g} \tau_{h}^{k}(\gamma)\left(t+\frac{k}{m} l_{g}\right)=\tau_{g}^{k}\left(\tau_{h}^{k}(\gamma)\right)(t) .
\end{aligned}
$$

Finally, one has smoothness of $\tau_{g}^{k}: \Omega_{\mathcal{K}} \rightarrow \Omega_{\mathcal{K}}$ for any fixed $g \in G$ since $\tau_{g}^{k}$ is linear:

$$
\begin{aligned}
\tau_{g}^{k}(\lambda \gamma+\eta)(t) & =D_{g}(\lambda \gamma+\eta)\left(t+\frac{k}{m} l_{g}\right) \\
& =\lambda D_{g} \gamma\left(t+\frac{k}{m} l_{g}\right)+D_{g} \eta\left(t+\frac{k}{m} l_{g}\right)=\lambda \tau_{g}^{k}(\gamma)+\tau_{g}^{k}(\eta)
\end{aligned}
$$

for all $\gamma, \eta \in W^{(\alpha+1) / 2,2}\left(\mathbb{R} / \mathbb{Z}, \mathbb{R}^{3}\right)$ and $\lambda \in \mathbb{R}$. In particular, for the differential of $\tau_{g}^{k}$ at $\gamma \in \Omega_{\mathcal{K}}$ one simply has

$$
\left(d \tau_{g}^{k}\right)_{\gamma}[\eta]=\tau_{g}^{k}(\eta) \text { for all } \eta \in W^{(\alpha+1) / 2,2}\left(\mathbb{R} / \mathbb{Z}, \mathbb{R}^{3}\right),
$$

which implies according to Definition 2.3 that $\Omega_{\mathcal{K}}$ is a smooth G-manifold, since $\tau_{g}^{k}$ is an isomorphism with inverse mapping

$$
\left(\tau_{g}^{k}\right)^{-1}(\gamma):=D_{-g} \gamma\left(t+\frac{k}{m} l_{-g}\right)
$$


where $l_{-g}$ is a representative of the group element $-g \in G$ (with $\left.g+(-g)=e:=[0] \in G\right)$, e.g. $l_{-g}=-l_{g}$.

For technical reasons we will have to reparametrize to arclength later in our existence proof of minimizers in the G-symmetric subset, and therefore we need to understand what kind of symmetry the arclength parametrization inherits from a symmetric curve.

Lemma 4.6. Let $m, k \in \mathbb{Z}, G=\mathbb{Z} /(m \mathbb{Z})$, and $\gamma: \mathbb{R} / \mathbb{Z} \rightarrow \mathbb{R}^{3}$ be an absolutely continuous curve with $\left|\gamma^{\prime}\right|>0$ a.e. and with length $\mathscr{L}(\gamma)=\mathrm{L} \in(0, \infty)$, such that for $\mathrm{g}=\left[\mathrm{l}_{\mathrm{g}}\right] \in \mathrm{G}$ the identity $\tau_{\mathrm{g}}^{\mathrm{k}}(\gamma)=\gamma$ holds with $\tau_{\mathrm{g}}^{\mathrm{k}}$ as in (29). Then the corresponding arclength parametrization $\Gamma \in \mathrm{C}^{0,1}\left(\mathbb{R} /(\mathrm{L} \mathbb{Z}), \mathbb{R}^{3}\right)$ satisfies

$$
\mathrm{D}_{\mathrm{g}} \Gamma\left(\mathrm{s}+\frac{\mathrm{k}}{\mathrm{m}} \mathrm{l}_{\mathrm{g}} \mathrm{L}\right)=\Gamma(\mathrm{s}) \text { for all } \mathrm{s} \in[0, \mathrm{~L}) .
$$

Since arclength reparametrizations of curves in $W^{(\alpha+1) / 2,2}$ inherit the same regularity as shown in the appendix in Lemma A.1 we immediately infer the following corollary.

Corollary 4.7. Let $\mathrm{m}, \mathrm{k} \in \mathbb{Z}$ and $\mathrm{G}=\mathbb{Z} /(\mathrm{m} \mathbb{Z})$ and let $\mathcal{K}$ be any knot class, and $\Omega_{\mathcal{K}}$ be the Banach manifold defined in Lemma 4.1] with G-symmetric subset $\Sigma_{\mathcal{K}}^{k}$ with respect to the group action given by $\tau^{\mathrm{k}}$ defined in $(\overline{29})$. Then, if $\gamma \in \Sigma_{\mathcal{K}}^{k}$ with length $\mathscr{L}(\gamma)=1$, its arclength parametrization $\Gamma: \mathbb{R} / \mathbb{Z} \rightarrow \mathbb{R}^{3}$ is contained in $\Sigma_{\mathcal{K}}^{k}$ as well.

Proof of Lemma 4.6. Differentiating the relation $\tau_{\mathrm{g}}^{\mathrm{k}}(\gamma)=\gamma$ with respect to t one obtains $\mathrm{D}_{g} \gamma^{\prime}\left(t+\frac{k}{m} l_{g}\right)=\gamma^{\prime}(t)$ for a.e. $t \in \mathbb{R} / \mathbb{Z}$. Since $D_{g} \in S O(3)$ we find that $\left|\gamma^{\prime}\right|$ is not only 1-periodic but also $\mathrm{kl}_{\mathrm{g}} / \mathrm{m}$-periodic, so that we can calculate for the arclength parameter

$$
\begin{aligned}
s\left(\frac{\mathrm{k}}{\mathrm{m}} \mathrm{l}_{\mathrm{g}}\right)=\int_{0}^{\frac{\mathrm{k}}{\mathrm{m}} l_{g}}\left|\gamma^{\prime}(\mathrm{t})\right| \mathrm{dt} & =\frac{1}{\mathrm{~m}} \int_{0}^{\mathrm{k} l_{g}}\left|\gamma^{\prime}(\mathrm{t})\right| \mathrm{dt} \\
& =\frac{\mathrm{k}}{\mathrm{m}} \mathrm{l}_{\mathrm{g}} \int_{0}^{1}\left|\gamma^{\prime}(\mathrm{t})\right| \mathrm{dt}=\mathrm{L} \frac{\mathrm{k}}{\mathrm{m}} \mathrm{l}_{\mathrm{g}},
\end{aligned}
$$

and therefore,

$$
\begin{gathered}
s\left(\frac{k}{m} l_{g}+t\right)=\int_{0}^{\frac{k}{m} l_{g}+t}\left|\gamma^{\prime}(\tau)\right| d \tau=\int_{0}^{\frac{k}{m} l_{g}}\left|\gamma^{\prime}(\tau)\right| d \tau+\int_{\frac{k}{m} l_{g}}^{\frac{k}{m} l_{g}+t}\left|\gamma^{\prime}(\tau)\right| d \tau \\
\stackrel{31}{=} L \frac{k}{m} l_{g}+\int_{0}^{t}\left|\gamma^{\prime}(\tau)\right| d \tau=L \frac{k}{m} l_{g}+s(t) .
\end{gathered}
$$

With $\Gamma(s(t))=\gamma(t)$ for all $t \in \mathbb{R} / \mathbb{Z}$ we infer from this by definition of the group action (29)

$$
\Gamma(s(t))=\gamma(t)=\tau_{g}^{k}(\gamma)(t) \stackrel{(29)}{=} D_{g} \gamma\left(t+\frac{k}{m} l_{g}\right)=D_{g} \Gamma\left(s\left(t+\frac{k}{m} l_{g}\right) \stackrel{(32)}{=} D_{g} \Gamma\left(s(t)+\frac{k}{m} l_{g} L\right) .\right.
$$

Now we turn our attention to torus knots. For relatively prime integers $\mathrm{a}, \mathrm{b} \in \mathbb{Z} \backslash\{0, \pm 1\}$ and some fixed $\rho \in(0,1)$ the curve

$$
\gamma_{\rho}(t):=\operatorname{Rot}(2 \pi a t)\left(\begin{array}{c}
1+\rho \cos (2 \pi b t) \\
0 \\
\rho \sin (2 \pi b t)
\end{array}\right)=\left(\begin{array}{c}
\cos (2 \pi a t)(1+\rho \cos (2 \pi b t) \\
\sin (2 \pi a t)(1+\rho \cos (2 \pi b t) \\
\rho \sin (2 \pi b t)
\end{array}\right) \text { for } t \in \mathbb{R} / \mathbb{Z}
$$


is a smooth representative of the torus knot class $\mathcal{T}(a, b)$. According to [9, Theorem 3.29] one has $\mathcal{T}(a, b)=\mathcal{T}(b, a)=\mathcal{T}(-a,-b)=\mathcal{T}(-b,-a)$. We can use the particular representative $\gamma_{\rho}$ defined in (33) to show that the G-symmetric subset of the Banach manifold $\Omega_{\mathcal{T}(a, b)}$ with respect to the group action (29) is not empty.

Lemma 4.8. Let $\alpha \in(2,3), a, b \in \mathbb{Z} \backslash\{0, \pm 1\}$ relatively prime, let $m \in \mathbb{N}, m>1$, divide $a$ or $\mathrm{b}$, and let $\mathrm{G}=\mathbb{Z} /(\mathrm{m} \mathbb{Z})$. Then the following is true: For any $\mathrm{k} \in \mathbb{Z} \backslash\{0\}$ with

$$
\begin{cases}{[a k+1]=e=[0] \in G} & \text { if } m \mid b \\ {[b k+1]=e=[0] \in G} & \text { if } m \mid a\end{cases}
$$

one has a nonempty G-symmetric subset

$$
\Sigma_{\mathrm{a}, \mathrm{b}}^{\mathrm{m}}:=\left\{\gamma \in \Omega_{\mathcal{T}(\mathrm{a}, \mathrm{b})}: \tau_{\mathrm{g}}(\gamma)=\gamma \text { for all } \mathrm{g} \in \mathrm{G}\right\},
$$

where $\tau_{g}$ is defined in (29).

Proof. It suffices to treat the case $m \mid b$. In Lemma A.5 in the appendix we show that such $k \in \mathbb{Z} \backslash\{0\}$ with (34) do exist, furthermore, $k$ is unique modulo m. Taking $\gamma_{\rho}$ as in (33) as a smooth and regular representative for $\mathcal{T}(a, b)$ that avoids the $z$-axis, we find that $\gamma_{\rho} \in \Omega_{\mathcal{T}(a, b)}$, and we directly compute

$$
\begin{aligned}
\tau_{g}\left(\gamma_{\rho}\right)(t) & =D_{g} \gamma_{\rho}\left(t+\frac{k}{m} l_{g}\right) \\
& =\operatorname{Rot}\left(2 \pi l_{g} / m\right) \operatorname{Rot}\left(2 \pi a\left(t+\frac{k}{m} l_{g}\right)\right)\left(\begin{array}{c}
1+\rho \cos \left(2 \pi b\left(t+\frac{k}{m} l_{g}\right)\right) \\
0 \\
\rho \sin \left(2 \pi b\left(t+\frac{k}{m} l_{g}\right)\right)
\end{array}\right) \\
& =\operatorname{Rot}\left(2 \pi a t+2 \pi(a k+1) l_{g} / m\right)\left(\begin{array}{c}
1+\rho \cos (2 \pi b t) \\
0 \\
\rho \sin (2 \pi b t)
\end{array}\right)=\gamma_{\rho}(t),
\end{aligned}
$$

where we used (34) in the argument of the last rotation. Hence, $\gamma_{\rho} \in \Sigma_{a, b}^{m}$.

Now we are ready to prove the existence of symmetric minimizers for the scaled O'Hara energy defined in (2) in the introduction. Notice that since $E_{\alpha}$ is continuously differentiable on the space of regular curves (see Remark 3.6), so is $S_{\alpha}$ since the length functional is continuously differentiable, even in the class of regular curves of class $W^{1,1}\left(\mathbb{R} / \mathbb{Z}, \mathbb{R}^{3}\right)$, and hence in particular on the Banach manifold $\Omega_{\mathcal{K}}$ for any (tame) knot class $\mathcal{K}$.

Theorem 4.9. Let $\alpha \in(2,3), a, b \in \mathbb{Z} \backslash\{0, \pm 1\}$ relatively prime, and let $m \in \mathbb{N}, m>1$, divide $\mathrm{a}$ or $\mathrm{b}$. Then for any $\mathrm{k} \in \mathbb{Z} \backslash\{0\}$ satisfying condition (34) of Lemma 4.8 there exists an arclength parametrized curve $\Gamma_{\min }^{\mathrm{m}} \in \Sigma_{\mathrm{a}, \mathrm{b}}^{\mathrm{m}} \subset \Omega_{\mathcal{T}(\mathrm{a}, \mathrm{b})}$ such that

$$
S_{\alpha}\left(\Gamma_{\min }^{\mathfrak{m}}\right)=\inf _{\Sigma_{a, b}^{\mathfrak{m}}} S_{\alpha}
$$

Here $\Sigma_{\mathrm{a}, \mathrm{b}}^{\mathrm{m}}$ is the nonempty $\mathrm{G}$-symmetric subset of $\Omega_{\mathcal{T}(\mathrm{a}, \mathrm{b})}, \mathrm{G}=\mathbb{Z} / \mathrm{m} \mathbb{Z}$, with respect to the group action of $\tau$ defined in (29); see Lemma 4.8

Proof. Without loss of generality we may assume $\mathrm{m} \mid \mathrm{b}$, the case $\mathrm{m} \mid \mathrm{a}$ can be treated analogously. According to Lemma 4.8 we have $\Sigma_{\mathrm{a}, \mathrm{b}}^{\mathrm{m}} \neq \emptyset$. The energy is finite on this set (see 
part (ii) of Theorem 3.2), so we find a minimizing sequence $\left(\gamma_{i}\right)_{i} \subset \Sigma_{a, b}^{m}$ with

$$
\lim _{i \rightarrow \infty} S_{\alpha}\left(\gamma_{i}\right)=\inf _{\Sigma_{a, b}^{\mathfrak{m}}} S_{\alpha} \in[0, \infty) \text {. }
$$

Since $S_{\alpha}$ is scale-invariant we may assume, in addition, that $\mathscr{L}\left(\gamma_{i}\right)=1$ for all $i \in \mathbb{N}$ (simply by scaling the $\gamma_{i}$ with scaling factor $\mathscr{L}\left(\gamma_{i}\right)^{-1}$ if necessary). In addition, by translations in the $z$-direction (thus keeping the symmetry), we may also assume that all $\gamma_{i}$ intersect the $x$ - $y$-plane.

By (36),

$$
S_{\alpha}\left(\gamma_{i}\right)=E_{\alpha}\left(\gamma_{i}\right) \leqslant C \text { for all } i \in \mathbb{N},
$$

where $C$ is a constant independent of $i$. Since $\mathscr{L}\left(\gamma_{i}\right)=1$ for all $i \in \mathbb{N}$, the corresponding arclength parametrizations $\Gamma_{i}$ all have the common domain $\mathbb{R} / \mathbb{Z}$ and $E_{\alpha}\left(\Gamma_{i}\right)=E_{\alpha}\left(\gamma_{i}\right)$ for all $i \in \mathbb{N}$. Moreover, according to (9) in part (i) of Theorem 3.2 these arclength parametrizations are all of class $W^{(\alpha+1) / 2,2}\left(\mathbb{R} / \mathbb{Z}, \mathbb{R}^{3}\right)$ satisfying

$$
\left[\Gamma_{i}^{\prime}\right]_{(\alpha-1) / 2,2} \leqslant 4^{4} \cdot 2^{2-2 \alpha} \mathrm{C} \text { for all } i \in \mathbb{N} \text {. }
$$

Since all $\Gamma_{i}$ have length 1 , each $\Gamma_{i}$ is contained in a closed ball $B_{i} \subset \mathbb{R}^{3}$ of radius $1 / 2$. All these closed balls $B_{i}$ must intersect the $x$-y-plane since $\Gamma_{i}$ does for each $i \in \mathbb{N}$. In addition, by symmetry the $B_{i}$ also intersect the $z$-axis. Indeed, the orbit of a point $x \in \Gamma_{i}$ under the action of $\mathrm{G}$ lies in a hyperplane orthogonal to the $z$-axis, and the convex hull of this orbit is an m-gon in that hyperplane that intersects the $z$-axis and is contained in $B_{i}$, so that $B_{i}$ itself intersects the $z$-axis as well. Therefore all $B_{i}$ and thus all $\Gamma_{i}(\mathbb{R} / \mathbb{Z})$ are contained in a cube of edge length 4 centered at the origin, so that

$$
\left\|\Gamma_{\mathrm{i}}\right\|_{\mathrm{L}^{\infty}} \leqslant \sqrt{8} \text { for all } i \in \mathbb{N} \text {. }
$$

Combining this with (37) and the identity $\left|\Gamma_{i}^{\prime}\right| \equiv 1$ for all $i \in \mathbb{N}$ we arrive at

$$
\left\|\Gamma_{i}\right\|_{W^{(\alpha+1) / 2,2}} \leqslant C_{1} \text { for all } i \in \mathbb{N} \text {, }
$$

where $C_{1}$ is independent of $i$. Together with the embedding inequality (12) we arrive at a uniform $C^{1,(\alpha / 2)-1}$-bound

$$
\left\|\Gamma_{i}\right\|_{C^{1,(\alpha / 2)-1}} \leqslant C_{E} C_{1} \text { for all } i \in \mathbb{N} \text {. }
$$

By the Arzela-Ascoli compactness theorem we find a subsequence (again denoted by $\Gamma_{i}$ ), which converges strongly in $C^{1}$ to a limit curve $\Gamma \in C^{1, \mu}$ for all $\mu \in(0,(\alpha / 2)-1)$. This convergence implies in particular that $\left|\Gamma^{\prime}\right| \equiv 1$. We have shown in Lemma 3.5 that $E_{\alpha}$ is lower semicontinuous even with respect to pointwise convergence, which implies that $E_{\alpha}(\Gamma) \leqslant \liminf _{i \rightarrow \infty} E_{\alpha}\left(\Gamma_{i}\right) \leqslant C$. According to Part (i) of Theorem 3.2 the limit curve $\Gamma$ is of class $W^{(\alpha+1) / 2,2}$ and injective. Now, the isotopy stability under $C^{1}$-convergence mentioned before (see [28] or [3]) gives $[\Gamma]=\left[\Gamma_{i}\right]=\mathcal{T}(a, b)$ for all $i \in \mathbb{N}$. In order to establish the symmetry of $\Gamma$ we use Corollary 4.7, which implies that

$$
\mathrm{D}_{\mathrm{g}} \Gamma_{\mathrm{i}}\left(s+\frac{\mathrm{k}}{\mathrm{m}} \mathrm{l}_{\mathrm{g}}\right)=\Gamma_{\mathrm{i}}(\mathrm{s}) \text { for all } s \in[0,1), i \in \mathbb{N} .
$$

Taking the limit $i \rightarrow \infty$ in this relation (for the subsequence $\Gamma_{i}$ converging in $C^{1}$ to $\Gamma$ ) implies

$$
\mathrm{D}_{\mathrm{g}} \Gamma\left(s+\frac{\mathrm{k}}{\mathrm{m}} l_{\mathrm{g}}\right)=\Gamma(\mathrm{s}) \text { for all } s \in[0,1)
$$

\footnotetext{
3 or even in a closed ball of radius $1 / 4$; see the short argument in [24].
} 
and hence $\tau_{g}(\Gamma)=\Gamma$ for all $g \in G$. Now, if there was some parameter $s \in \mathbb{R} / \mathbb{Z}$ such that $\left(\Gamma^{1}(\mathrm{~s})\right)^{2}+\left(\Gamma^{2}(\mathrm{~s})\right)^{2}=0$ we could apply $(38)$ to find that

$$
\Gamma\left(s+\frac{k}{m} l_{g}\right)=\Gamma(s) \text { for all } g \in G,
$$

since the rotation $D_{g}=\operatorname{Rot}\left(2 \pi l_{g} / m\right)$ about the $z$-axis and hence also its inverse leave every point on the $z$-axis fixed. But (39) contradicts the injectivity of $\Gamma$ since $k \neq 0$ and $\mathrm{g} \in \mathrm{G}$ may be chosen to be non-trivial. Thus we have shown that $\Gamma \in \Sigma_{\mathrm{a}, \mathrm{b}}^{\mathrm{m}} \subset \Omega_{\mathcal{T}(\mathrm{a}, \mathrm{b})}$. This together with the lower semicontinuity of $E_{\alpha}$ established in Lemma 3.5 finally implies minimality for $\Gamma_{\min }^{\mathrm{m}}:=\Gamma$ because

$$
\inf _{\Sigma_{a, b}^{\mathfrak{m}}} S_{\alpha} \leqslant S_{\alpha}(\Gamma)=E_{\alpha}(\Gamma) \leqslant \liminf _{i \rightarrow \infty} E_{\alpha}\left(\Gamma_{i}\right)=\lim _{i \rightarrow \infty} S_{\alpha}\left(\Gamma_{i}\right)=\inf _{\Sigma_{a, b}^{\mathfrak{m}}} S_{\alpha}
$$

Now we can convince ourselves that these symmetric minimizing torus knots are all critical for the scaled energy functional $S_{\alpha}$ on all of $\Omega_{\mathcal{T}(a, b)}$.

Corollary 4.10. Any of the minimizing torus knots $\Gamma_{\mathrm{min}}^{\mathrm{m}} \in \Sigma_{\mathrm{a}, \mathrm{b}}^{\mathrm{m}}$ found in Theorem 4.9 are critical points of the scaled energy $S_{\alpha}=\mathscr{L}^{\alpha-2} \mathrm{E}_{\alpha}$ and therefore of class $C^{\infty}\left(\mathbb{R} / \mathbb{Z}, \mathbb{R}^{3}\right)$.

Proof. We have seen in Corollary 4.2 that $\Omega_{\mathcal{T}(\mathrm{a}, \mathrm{b})}$ is a smooth manifold modeled over the Banach space $W^{(\alpha+1) / 2,2}\left(\mathbb{R} / \mathbb{Z}, \mathbb{R}^{3}\right)$. In addition, according to Lemma $4.4 \Omega_{\mathcal{T}(\mathrm{a}, \mathrm{b})}$ is even a smooth $G$-manifold under the action of the finite group $G:=\mathbb{Z} /(m \mathbb{Z})$ for $m \in \mathbb{N} \backslash\{1\}$. Moreover, the scaled energy $S_{\alpha}=\mathscr{L}^{\alpha-2} \mathrm{E}_{\alpha}$ is of class $\mathrm{C}^{1}$ on an open subset of the Banach space $W^{(\alpha+1) / 2,2}\left(\mathbb{R} / \mathbb{Z}, \mathbb{R}^{3}\right)$ containing $\Omega_{\mathcal{T}(a, b)}$ as mentioned in Remark 3.6 , and $S_{\alpha}$ is invariant under the action of $\tau$ since rotations in the ambient space and parameter shifts obviously do not alter the energy value; see Remark 1.1. Since the $\Gamma_{\min }^{\mathrm{m}}$ minimize $S_{\alpha}$ in $\Sigma_{a, b}^{m}$, they are $\left.S_{\alpha}\right|_{\Sigma_{a, b}^{m}}$-critical and therefore, according to Palais' Theorem 2.4, the $\Gamma_{\min }^{\mathrm{m}}$ are also critical for $S_{\alpha}$ on the full domain $\Omega_{\mathcal{T}(a, b)}$. The smoothness now follows by the regularity theorem of Blatt and Reiter mentioned in Remark 3.6 .

In order to show that there are at least two $S_{\alpha}$-critical knots in every non-trivial torus knot class $\mathcal{T}(a, b)$ we recall the definition of periodicity of knots from [9, p. 256] (see also [23, Definition 8.3]): Any curve $\gamma \in \mathrm{C}^{0}\left(\mathbb{R} / \mathbb{Z}, \mathbb{R}^{3}\right)$ being injective on $[0,1)$ that does not intersect the $z$-axis, and for which there is an integer $\mathrm{q} \in \mathbb{N} \backslash\{1\}$ such that

$$
\operatorname{Rot}(2 \pi / q) \gamma(\mathbb{R} / \mathbb{Z})=\gamma(\mathbb{R} / \mathbb{Z})
$$

has period q, or is q-periodic.

For torus knots the possible periods are known; see [9, Proposition 14.27]:

Theorem 4.11. If $\mathrm{q} \in \mathbb{N} \backslash\{1\}$ is a period of a curve $\gamma \in \mathrm{C}^{0}\left(\mathbb{R} / \mathbb{Z}, \mathbb{R}^{3}\right)$ with $[\gamma]=\mathcal{T}(\mathrm{a}, \mathrm{b})$ for relatively prime integers $\mathrm{a}, \mathrm{b} \in \mathbb{Z} \backslash\{0, \pm 1\}$, then $\mathrm{q} \mid \mathrm{a}$ or $\mathrm{q} \mid \mathrm{b}$. Conversely, if $\mathrm{q} \in \mathbb{N} \backslash\{1\}$ divides $\mathrm{a}$ or $\mathrm{b}$, then there is a representative $\gamma \in \mathrm{C}^{0}\left(\mathbb{R} / \mathbb{Z}, \mathbb{R}^{3}\right)$ such that $\mathrm{q}$ is a period of $\gamma$.

This result allows us to prove that there are at least two $S_{\alpha}$-critical knots in every torus knot class, which is our central result, Theorem 1.2 mentioned in the introduction. 
Proof of Theorem 1.2 For each $m \in \mathbb{N} \backslash\{1\}$ dividing a or $b$, and for each $k \in \mathbb{Z}$ satisfying (34) Theorem 4.9 in connection with Corollary 4.10 gives us at least one arclength parametrized curve

$$
\Gamma_{\min }^{\mathrm{m}} \in \Sigma_{\mathrm{a}, \mathrm{b}}^{\mathrm{m}} \cap \mathrm{C}^{\infty}\left(\mathbb{R} / \mathbb{Z}, \mathbb{R}^{3}\right)
$$

that is $S_{\alpha}$-critical. Choosing $m_{1}:=a$ and $k_{1}$ such that $k_{1}$ satisfies (34) for $m=m_{1}$, as well as $m_{2}:=b$ and $k_{2}$ satisfying (34) for $m=m_{2}$, we obtain two curves

$$
\Gamma_{1}:=\Gamma_{\min }^{\mathrm{a}} \in \Sigma_{\mathrm{a}, \mathrm{b}}^{\mathrm{a}} \cap \mathrm{C}^{\infty}\left(\mathbb{R} / \mathbb{Z}, \mathbb{R}^{3}\right) \text { and } \Gamma_{2}:=\Gamma_{\min }^{\mathrm{b}} \in \Sigma_{\mathrm{a}, \mathrm{b}}^{\mathrm{b}} \cap \mathrm{C}^{\infty}\left(\mathbb{R} / \mathbb{Z}, \mathbb{R}^{3}\right)
$$

with

$$
\begin{aligned}
& D_{g} \Gamma_{1}(\mathbb{R} / \mathbb{Z})=\Gamma_{1}(\mathbb{R} / \mathbb{Z}) \text { for all } g \in \mathbb{Z} /(\mathrm{a} \mathbb{Z}), \\
& \mathrm{D}_{\mathrm{h}} \Gamma_{2}(\mathbb{R} / \mathbb{Z})=\Gamma_{2}(\mathbb{R} / \mathbb{Z}) \text { for all } \mathrm{h} \in \mathbb{Z} /(\mathrm{b} \mathbb{Z})
\end{aligned}
$$

by means of (30) with $L=1$ for $m=m_{1}=a$, and for $m=m_{2}=b$, respectively.

Any isometry I : $\mathbb{R}^{3} \rightarrow \mathbb{R}^{3}$ can be written as $\mathrm{I}(x)=\mathrm{O} x+\xi, x \in \mathbb{R}^{3}$, for some orthogonal matrix $O \in O(3)$ and some vector $\xi \in \mathbb{R}^{3}$. Since the orthogonal group $O(3)$ is the semidirect product of $\mathrm{SO}(3)$ and $\mathrm{O}(1)$ [14, p.50], we can write $\mathrm{O}=\mathrm{SR}$ for some rotation $\mathrm{R} \in \mathrm{SO}(3)$ and some $S \in \mathrm{O}(1)$, and the latter may be a reflection across one two-dimensional subspace $E \subset \mathbb{R}^{3}$, or else $S$ is the identity mapping. But if $S$ is a reflection and we assume that

$$
\mathrm{I} \circ \Gamma_{1}(\mathbb{R} / \mathbb{Z})=\Gamma_{2}(\mathbb{R} / \mathbb{Z}),
$$

then (since translations and rotations do not alter the knot class)

$$
\mathcal{T}(\mathrm{a}, \mathrm{b})=\left[\Gamma_{2}\right]=\left[\mathrm{I} \circ \Gamma_{1}\right]=\left[\mathrm{O} \Gamma_{1}\right]=\left[S R \Gamma_{1}\right] \neq\left[\mathrm{R} \Gamma_{1}\right]=\left[\Gamma_{1}\right]=\mathcal{T}(\mathrm{a}, \mathrm{b}),
$$

which is a contradiction. To justify the inequality in (43) note that according to [9, Theorem 3.29] the torus knot class $\mathcal{T}(a, b)$ is not amphichiral, i.e., the reflection $S \gamma$ of any curve $\gamma$ with $[\gamma]=\mathcal{T}(a, b)$ at some two-dimensional subspace $E \subset \mathbb{R}^{3}$ would represent the different torus knot class $\mathcal{T}(a,-b) \neq \mathcal{T}(a, b)$; see [9, Prop. 3.27]. So, the assumption (42) necessarily leads to the representation $\mathrm{I}(x)=\mathrm{R} x+\xi, x \in \mathbb{R}^{3}$, for some rotation $\mathrm{R} \in \mathrm{SO}(3)$ (about some axis through the origin) and some translational vector $\xi \in \mathbb{R}^{3}$.

This together with (40), (41), and the fact that $\Gamma_{1}, \Gamma_{2} \in \Omega_{\mathcal{T}(\mathrm{a}, \mathrm{b})}$ both do not intersect the $z$-axis, implies under the assumption (42) that $\Gamma_{2}=\mathrm{I} \circ \Gamma_{1}$ is a-periodic with respect to the axis $I\left(\mathbb{R e}_{3}\right)$ in addition to being b-periodic with respect to the $z$-axis; see also Lemma A.7 in the appendix. Theorem 4.12 below then implies that the axis $I\left(\mathbb{R e}_{3}\right)$ coincides with the $z$-axis since the two rotational axes must necessarily intersect, and if there were only one intersection point of these axes, then the two different rotational angles $2 \pi / a \neq 2 \pi / b$ would lead to a nonempty intersection of $\Gamma_{2}$ with one of the rotational axes contradicting the periodicity of $\Gamma_{2}$; see Part (1)(iii) of Theorem 4.12 .

Since the $z$-axis equals its image under the isometry I we can infer in particular that the vector $\xi=R 0+\xi=I(0)$ is contained in the $z$-axis; hence $\xi=\left(0,0, \xi_{3}\right)$. Therefore, we find some $\lambda \in \mathbb{R}$ such that the point $I\left(e_{3}\right)=\mathrm{Re}_{3}+\xi_{3} e_{3}$ which is also contained in the $z$-axis may be written as $I\left(e_{3}\right)=\lambda e_{3}$ so that $\operatorname{Re}_{3}=\left(\lambda-\xi_{3}\right) e_{3}=: \mu e_{3}$. So $\mu$ is a real eigenvalue for the rotation $\mathrm{R} \in \mathrm{SO}(3)$; hence $\mu$ is either +1 or -1 . In the first case $e_{3}$ belongs to the fixed point set of $R$ which implies that $R$ is a rotation about the $z$-axis. If $\mu=-1$, on the other hand, $\mathrm{R}$ is a rotation about an axis perpendicular to the $z$-axis with the rotational angle $\pi$. 
In both cases $R$ commutes with $D_{h}$ on $\Gamma_{1}$, see Lemma A.8, which itself is a rotation about the $z$-axis, so that we infer (omitting the domain $\mathbb{R} / \mathbb{Z}$ in each term)

$$
R \Gamma_{1}+\xi \stackrel{(42)}{=} \Gamma_{2} \stackrel{(41)}{=} D_{h} \Gamma_{2} \stackrel{(42)}{=} D_{h}\left(R \Gamma_{1}+\xi\right)=D_{h} R \Gamma_{1}+D_{h}(\xi)=D_{h} R \Gamma_{1}+\xi=R D_{h} \Gamma_{1}+\xi,
$$

where the second to last equality is due to the fact that $\xi$ is contained in the $z$-axis. This leads to

$$
\mathrm{R} \Gamma_{1}(\mathbb{R} / \mathbb{Z})=\mathrm{RD}_{\mathrm{h}} \Gamma_{1}(\mathbb{R} / \mathbb{Z}),
$$

which implies a second symmetry of $\Gamma_{1}$ in addition to (40):

$$
\mathrm{D}_{\mathrm{h}} \Gamma_{1}(\mathbb{R} / \mathbb{Z})=\Gamma_{1}(\mathbb{R} / \mathbb{Z}) \text { for all } h \in \mathbb{Z} /(\mathrm{b} \mathbb{Z}) .
$$

Now choosing $g=[1] \in \mathbb{Z} /(a \mathbb{Z})$ and $h=[1] \in \mathbb{Z} /(b \mathbb{Z})$ we find another period of $\Gamma_{1}$ as follows (again omitting the domain $\mathbb{R} / \mathbb{Z}$ in each term):

$$
\Gamma_{1} \stackrel{(40)}{=} D_{g} \Gamma_{1} \stackrel{(44)}{=} D_{g} D_{h} \Gamma_{1}=\operatorname{Rot}\left(\frac{2 \pi}{a}\right) \operatorname{Rot}\left(\frac{2 \pi}{b}\right) \Gamma_{1}=\operatorname{Rot}\left(\frac{2 \pi}{a b} \cdot(b+a)\right) \Gamma_{1} .
$$

The two integers, $(a+b)$ and $a b$, are relatively prime (see Lemma A.6 in the appendix), so that $(a+b)$ is invertible modulo $a b$, which means that we can find some integer $k \in \mathbb{Z}$ such that $k(a+b) \equiv 1 \bmod a b$. This implies by means of (45) that

$$
\Gamma_{1} \stackrel{(45)}{=}\left[\operatorname{Rot}\left(\frac{2 \pi}{a b} \cdot(a+b)\right)\right]^{k} \Gamma_{1}=\operatorname{Rot}\left(\frac{2 \pi}{a b} \cdot k(a+b)\right) \Gamma_{1}=\operatorname{Rot}\left(\frac{2 \pi}{a b}\right) \Gamma_{1} .
$$

In other words, $\Gamma_{1}$ is $(a b)$-periodic, which contradicts Theorem 4.11, since ab divides neither $a$ nor $b$. This is the final contradiction and concludes the proof of the theorem.

Essential for the previous proof is the following result on possible rotational symmetries of general non-trivial knots. Most of these facts can also be extracted from Grünbaum and Shephard's classification of possible symmetry groups of knots [19] in combination with their characterization of finite subgroups of $\mathrm{O}(3)$ in [18]. Here we present a purely geometrical approach, adding information about possible periods of a knot.

Theorem 4.12 (Rotational symmetries of knots). If a non-trivial tame knot $\Gamma$ has a rotational symmetry about an axis $v$ with angle $\varphi \in(-\pi, \pi]$ and $\Gamma \cap \nu \neq \emptyset$, then $\varphi=\pi$. If $\Gamma$ has two axes $\nu_{1}$ and $\nu_{2}$ of rotational symmetry with respect to rotation angles $\varphi_{1}=\frac{2 \pi}{a_{1}}, \varphi_{2}=\frac{2 \pi}{a_{2}}$ for some integers $a_{1}, a_{2} \geqslant 2$, then $v_{1} \cap v_{2} \neq \emptyset$.

Furthermore, if $v_{1} \cap v_{2}=\{\mathrm{p}\}$ for some $\mathrm{p} \in \mathbb{R}^{3}$, the following holds.

(1) For $\varphi_{1} \neq \varphi_{2}$ we have

(i) $v_{1} \perp v_{2}$;

(ii) Either $a_{1}=2$ and $a_{2} \geqslant 3$, or vice versa;

(iii) If $\mathrm{a}_{1}=2$ in Part (ii) then $v_{1} \cap \Gamma \neq \emptyset$ and $v_{2} \cap \Gamma=\emptyset$. If $\mathrm{a}_{2}=2$ in Part (ii) then $v_{2} \cap \Gamma \neq \emptyset$ and $v_{1} \cap \Gamma=\emptyset$.

(2) If $\varphi_{1}=\varphi_{2}$, then we have $\varphi_{1}=\varphi_{2}=\pi$.

Before proving this theorem let us provide a slight generalization of a result of Grünbaum and Shephard [19, Lemma 1] whose paper actually motivated our purely geometric proof of Theorem 4.12 .

Lemma 4.13. For $a \in \mathbb{N}, a \geqslant 3$, a knot cannot have more than one axis of rotational symmetry with rotational angle $2 \pi / a$. 
Proof. Assume that there are two axes $v$ and $w$ (not necessarily through the origin) of rotational symmetry for a $\operatorname{knot} \Gamma \in \mathbb{R}^{3}$ with respect to the rotational angle $\beta:=2 \pi / \mathrm{a}$ for some integer $a \geqslant 3$. Fix a point $x \in \Gamma$ and look at its orbit

$$
\mathrm{O}_{v}:=\left\{x, x_{1}, x_{2}, \ldots, x_{a-1}\right\} \subset \Gamma
$$

under the action of the rotation $\operatorname{Rot}(\beta, v)$, i.e., $x_{i}:=\operatorname{Rot}(\beta i, v) x$ for $i=1, \ldots, a-1$, where the symbol $\operatorname{Rot}(\beta, v)$ denotes the rotation about the axis $v$ with angle $\beta$. The points in $\mathrm{O}_{v}$ are separated on $\Gamma$ by subarcs of length $\mathscr{L}(\Gamma) /$ a, and those points form a regular a-gon spanning an affine plane $E_{v}$ perpendicular to the axis $v$ since $a \geqslant 3$. Let

$$
\mathrm{O}_{w}:=\left\{x, \xi_{1}, \xi_{2}, \ldots, \xi_{\mathrm{a}-1}\right\} \subset \Gamma
$$

be the corresponding orbit of $x$ under the rotation $\operatorname{Rot}(\beta, w)$, which also forms a regular agon spanning an affine plane $E_{w}$ perpendicular to the other axis $w$. The points in $O_{w}$ are separated on $\Gamma$ by subarcs of length $\mathscr{L}(\Gamma) / \mathrm{a}$ as well, so that either $x_{i}=\xi_{i}$, or $x_{i}=\xi_{a-1-i}$ for $i=1, \ldots, a-1$. In both cases the regular a-gons coincide, as well as the affine planes $E_{v}$ and $E_{w}$. Hence $v$ and $w$ are parallel, and since both axes of rotational symmetry must intersect the midpoint of the a-gon

$$
\left(x+x_{1}+x_{2}+\cdots+x_{a-1}\right) / a=\left(x+\xi_{1}+\xi_{2}+\cdots+\xi_{a-1}\right) / a,
$$

the axes $v$ and $w$ must coincide.

Proof of Theorem 4.12 To prove the first assertion, consider an angle $\varphi$ of an arbitrary rotation about an axis $v$ with $v \cap \Gamma \neq \emptyset$ with $\varphi \neq \pi$. Then we have $2 \pi /|\varphi|>2$ arcs entering $x \in v \cap \Gamma$. But then $\Gamma$ is not embedded. Hence, if $\varphi \neq \pi$, we need to have $v \cap \Gamma=\emptyset$.

Now we consider the case of rotational symmetry about two different axes. We start by showing that a knot cannot have two rotational symmetry axes which are disjoint, no matter which angles are considered.

To that extent, assume $\Gamma$ has two rotational symmetry axes $v_{1}, v_{2}$ with rotational angles $\varphi_{1}=2 \pi / a_{1}$ and $\varphi_{2}=2 \pi / a_{2}$ for some integers $a_{1}, a_{2} \geqslant 2$, such that $\nu_{1} \cap \nu_{2}=\emptyset$. If $a_{1}=a_{2}=2$ we argue as follows. Consider the two parallel affine planes $E_{1}, E_{2} \subset \mathbb{R}^{3}$ such that $v_{1} \subset \mathrm{E}_{1}$ and $v_{2} \subset \mathrm{E}_{2}$, and $\mathrm{d}:=\operatorname{dist}\left(\mathrm{E}_{1}, \mathrm{E}_{2}\right)>0$. Then $\Gamma$ cannot be fully contained in the closed infinite slab

$$
S:=\left\{x \in \mathbb{R}^{3}: \operatorname{dist}\left(x, E_{i}\right) \leqslant d \text { for } i=1,2\right\},
$$

since any point in $S$ gets mapped into the exterior $\mathbb{R}^{3} \backslash S$ by at least one of the rotations $\operatorname{Rot}\left(\pi, v_{i}\right), i=1,2$. Now without loss of generality we may assume that $E_{1}$ and $E_{2}$ are parallel to the $x-y$-plane, i.e.,

$$
E_{i}:=\left\{y=\left(y^{1}, y^{2}, y^{3}\right) \in \mathbb{R}^{3}: y^{3}=R_{i}\right\} \text { for } i=1,2
$$

with $R_{1}>R_{2}$, and we denote the curve points with the largest and the smallest $z$ coordinate by $x_{\max } \in \Gamma$ and $x_{\min } \in \Gamma$, respectively. We may assume without loss of generality that

$$
\operatorname{dist}\left(x_{\max }, S\right) \geqslant \operatorname{dist}\left(x_{\min }, S\right)
$$

and deduce for the point $x^{*}:=\operatorname{Rot}\left(\pi, v_{2}\right) x_{\max }$ by means of (46) the identity

$$
\operatorname{dist}\left(x^{*}, E_{2}\right)=\operatorname{dist}\left(x_{\max }, E_{2}\right)=\operatorname{dist}\left(x_{\max }, S\right)+d \stackrel{(46)}{\geqslant} \operatorname{dist}\left(x_{\min }, S\right)+d>\operatorname{dist}\left(x_{\min }, S\right) \text {. }
$$


Therefore, $x^{*}$ has a strictly smaller $z$-coordinate than $x_{\min }$ since $x^{*}$ lies in $\mathbb{R}^{3} \backslash S$ below the lower affine plane $E_{2}$, which contradicts the minimality of $x_{\min }$. This settles the case $a_{1}=a_{2}=2$.

If, say $a_{1} \geqslant 3$ and $a_{2} \geqslant 2$, we can apply repeatedly Lemma A.7 in the appendix to the set $M:=\Gamma$ and to the isometry I defined as the rotation about $\nu_{2}$ with respect to the rotational angle $\varphi_{2}=2 \pi / a_{2}$. The fact that $I(\Gamma)=\Gamma$ because of the rotational symmetry of $\Gamma$ with respect to the rotation about $\nu_{2}$, together with (70) allows us to find new symmetry axes for $\Gamma$ by rotating $v_{1}$ about the other axis $v_{2}$. That is, all axes

$$
v_{1}^{i}=\operatorname{Rot}\left(\frac{2 \pi \cdot i}{a_{2}}, v_{2}\right) v_{1}, \quad i=0, \ldots, a_{2}-1
$$

are axes of rotational symmetry for $\Gamma$ with rotational angle $\varphi_{1}=\frac{2 \pi}{a_{1}}$, where, as before, the symbol $\operatorname{Rot}(\beta, w)$ denotes the rotation about an axis $w$ with rotational angle $\beta \in \mathbb{R}$. Since $a_{2} \geqslant 2$ and $v_{1} \cap v_{2}=\emptyset$, there are now at least two different axes of rotational symmetry with respect to the angle $\varphi_{1}=2 \pi / a_{1}$, contradicting Lemma 4.13. Thus we have shown that $v_{1} \cap v_{2} \neq \emptyset$.

We will now assume that $v_{1} \cap v_{2}=\{p\}$ for some $p \in \mathbb{R}^{3}$. Without loss of generality we may restrict to the case $p=0$ because of translational invariance of the remaining claims. The corresponding rotational angles are $\varphi_{1}=\frac{2 \pi}{a_{1}}$ and $\varphi_{2}=\frac{2 \pi}{a_{2}}$ for some integers $a_{1}, a_{2} \geqslant 2$. To prove Part (1) we take $a_{1} \neq a_{2}$ and consider the possible combinations of $a_{1}$ and $a_{2}$.

1. $a_{1}, a_{2} \geqslant 3$.

In this case both rotational angles are contained in $(0, \pi)$ so that the first part of the theorem implies that $\Gamma$ is disjoint from both axes $v_{1}$ and $v_{2}$. As before, we may construct copies of $v_{1}$ such that $\Gamma$ is rotational symmetric with respect to the axis $v_{1}$, as well as to its copies

$$
v_{1}^{i}=\operatorname{Rot}\left(\frac{2 \pi \cdot i}{a_{2}}, v_{2}\right) v_{1}, \quad i=0, \ldots, a_{2}-1 .
$$

In other words, all these lines are axes of rotational symmetry for $\Gamma$ with the same rotational angle $\varphi_{1}=\frac{2 \pi}{a_{1}}$ with $a_{1} \geqslant 3$, and there are at least two of those since $a_{2} \geqslant 3$, contradicting Lemma 4.13. Thus, either $a_{1}=2$ and $a_{2} \geqslant 3$, or $a_{2}=2$ and $a_{1} \geqslant 3$ which proves Part (1)(ii). Furthermore, the presented argument implies Part (2).

2. $a_{1} \geqslant 3, a_{2}=2$, (the case $a_{1}=2$ and $a_{2} \geqslant 3$ can be treated analogously).

In this case, we will have to take into account the angle $\Varangle\left(v_{1}, v_{2}\right)=: \alpha \in(0, \pi / 2]$. Assume that $0<\alpha<\pi / 2$. Then we may construct a second rotational symmetry axis for $\Gamma$ with rotational angle $\varphi_{1}=\frac{2 \pi}{a_{1}}$, namely

$$
v_{1}^{1}=\operatorname{Rot}\left(\pi, v_{2}\right) v_{1}
$$

Notice that

$$
\Varangle\left(v_{1}, v_{1}^{1}\right)=\min \{2 \alpha, \pi-2 \alpha\} \in(0, \pi / 2],
$$

so that in particular $v_{1}^{1} \neq v_{1}$. So, there are two distinct axes of rotational symmetry for $\Gamma$ with rotational angle $2 \pi / a_{1}$ with $a_{1} \geqslant 3$, contradicting Lemma 4.13 again. Therefore, we have $v_{1} \perp v_{2}$, which is (1)(i).

Since the first part of the theorem already implies that $\Gamma \cap \nu_{1}=\emptyset$ because $\varphi_{1} \in(0, \pi)$ it suffices to show $v_{2} \cap \Gamma \neq \emptyset$ to finally establish Part (1)(iii).

Assume that $v_{2} \cap \Gamma=\emptyset$, then both axes $v_{1}$ and $v_{2}$ are disjoint from $\Gamma$. Then the rotational symmetry is a periodicity, see [9, p. 256]. We denote by $L:=\mathscr{L}(\Gamma)$ the length of $\Gamma$. The 
plane $\mathrm{H}:=v_{1}^{\perp}$ contains $v_{2}$ according to Part (1)(i), and we immediately deduce that $\mathrm{H} \cap \Gamma \neq$ $\emptyset$ because of the periodicity about $\nu_{2}$. Fix a point $x_{0} \in \mathrm{H} \cap \Gamma$, and look at its orbit

$$
\mathrm{O}_{v_{1}}:=\left\{\mathrm{x}_{0}, \ldots, \mathrm{x}_{\mathrm{a}_{1}-1}\right\} \subset \mathrm{H} \cap \Gamma
$$

under the action of the rotation Rot $\left(\varphi_{1}, v_{1}\right)$ but now - in contrast to the proof of Lemma 4.13 - labelled according to the corresponding arclength parameters. That is, $x_{i}=\Gamma\left(s_{i}\right)$ for $i=0, \ldots, a_{1}-1$ such that $0 \leqslant s_{0}<s_{1}<\ldots<s_{a_{1}-1}<L$, and there exists $k \in \mathbb{N}$ with $\operatorname{gcd}\left(k, a_{1}\right)=1$ and unique modulo $a_{1}$, such that

$$
x_{i}=\operatorname{Rot}\left(\frac{2 \pi \cdot k i}{a_{1}}, v_{1}\right) x_{0}, \quad i=0, \ldots, a_{1}-1 .
$$

To justify this, observe first that periodicity of $\Gamma$ implies that the subarcs on $\Gamma$ connecting consecutive $x_{i}$ have equal length, i.e., $s_{i+1}-s_{i}=L / a_{1}$ for all $i=0, \ldots, a_{1}-1$, and in general

$$
s_{j}-s_{i}=\frac{L}{a_{1}}(j-i) \quad 0 \leqslant i \leqslant j \leqslant a_{1}-1 .
$$

Reordering the points in the orbit $\mathrm{O}_{v_{1}}$ according to the rotation counterclockwise, starting at $y_{0}:=x_{0}$ leads to $\left\{y_{0}, \ldots, y_{a_{1}-1}\right\}$ defined as $y_{j}:=\operatorname{Rot}\left(2 \pi j / a_{1}, v_{1}\right) y_{0}$. There is an integer $\mathrm{m} \in\left\{1, \ldots, a_{1}-1\right\}$ such that $y_{1}=\Gamma\left(s_{m}\right)=x_{m}$, so the oriented subarc on $\Gamma$ starting at $x_{0}=y_{0}$ with endpoint $y_{1}=x_{m}$ has length $s_{m}-s_{0}=m L / a_{1}$ by means of (48). The same holds true for every oriented subarc from $y_{j}$ to $y_{j+1}$ for $j=1, \ldots, a_{1}-1$, so that we arrive at the general relation

$$
x_{[j \cdot m]}=\Gamma\left(s_{[j \cdot m]}\right)=y_{j}=\operatorname{Rot}\left(\frac{2 \pi j}{a_{1}}, v_{1}\right) y_{0}=\operatorname{Rot}\left(\frac{2 \pi j}{a_{1}}, v_{1}\right) x_{0}, \quad j=1, \ldots, a_{1}-1,
$$

where we denoted $[j \cdot m]=j \cdot m \bmod a_{1}$. If we had $\operatorname{gcd}\left(m, a_{1}\right)>1$ then the least common multiple $\operatorname{lcm}\left(m, a_{1}\right)$ of $m$ and $a_{1}$ could be written as $\operatorname{lcm}\left(m, a_{1}\right)=m \cdot a_{1} / \operatorname{gcd}\left(m, a_{1}\right)=: m$. $n$, where $1<n<a_{1}-1$ is a positive integer. Thus, $n \cdot m=0 \bmod a_{1}$, so that (49) implies $x_{[n \cdot m]}=\Gamma\left(s_{0}\right)=y_{n}$. But this would mean that the remaining points $y_{n+1}, \ldots, y_{a_{1}-1}$ would not be in the orbit $\mathrm{O}_{v_{1}}$ under the rotation, which is a contradiction.

Hence $\operatorname{gcd}\left(m, a_{1}\right)=1$ so that $m$ possesses an inverse modulo $a_{1}$, i.e., there is a unique $k \in\left\{1, \ldots, a_{1}-1\right\}$ such that $k \cdot m=1 \bmod a_{1}$. Inserting this into (49) we obtain $x_{[j \cdot m]}=$ $\operatorname{Rot}\left(\frac{2 \pi j \cdot m \cdot k}{a_{1}}, v_{1}\right) x_{0}$ for $j=1, \ldots, a_{1}-1$. Given any $i \in\left\{1, \ldots, a_{1}-1\right\}$ we choose $j:=i \cdot k$ to finally obtain (47).

As $\Gamma$ is 2-periodic around $v_{2} \subset \mathrm{H}$, there exist $\bar{x}_{i}=\Gamma\left(\bar{s}_{i}\right)=\in \Gamma \cap \mathrm{H}$ such that

$$
\bar{x}_{i}=\operatorname{Rot}\left(\pi, v_{2}\right) x_{i}, \quad i=0, \ldots, a_{1}-1 .
$$

In terms of arclength on $\Gamma$ we find $\left|s_{i}-\bar{s}_{i}\right|=L / 2$ for each $i=0, \ldots, a_{1}-1$.

By a short calculation, e.g., by means of the matrix representations of $\operatorname{Rot}\left(\pi, v_{2}\right)$ and Rot $\left(2 \pi k i / a_{1}, v_{1}\right)$ with respect to an orthonormal basis containing the unit vectors through $v_{1}$ and $v_{2}$, we arrive at

$$
\bar{x}_{i}=\operatorname{Rot}\left(\frac{2 \pi \cdot k(-i)}{a_{1}}, v_{1}\right) \bar{x}_{0}, \quad i=0, \ldots, a_{1}-1 .
$$

Next, we consider the circle $S:=\partial B_{r}(0) \cap H$ with $r:=\operatorname{dist}\left(x_{0}, 0\right)$. We have $x_{i}, \bar{x}_{i} \in S$ for all $i=0, \ldots, a_{1}-1$. We are going to determine the order of these points on $S$, and 
consider first only the $x_{i}$. Due to the $a_{1}$-periodicity, there is a unique successor $x_{i_{k}}$ of $x_{0}$ (counterclockwise) on $S$ which has a distance of $2 \pi r / a_{1}$ to $x_{0}$ on $S$ and is defined by (47):

$$
x_{i_{k}}=\operatorname{Rot}\left(\frac{2 \pi \cdot k i_{k}}{a_{1}}, v_{1}\right) x_{0}=\operatorname{Rot}\left(\frac{2 \pi \cdot 1}{a_{1}}, v_{1}\right) x_{0}
$$

which is equivalent to $k i_{k} \equiv a_{1} 1$. Thus $i_{k}$ is the unique inverse of $k$ in $\mathbb{Z} / a_{1} \mathbb{Z}$ which exists as $\operatorname{gcd}\left(k, a_{1}\right)=1$. Repeating this argument for the other successors, we arrive at the order

$$
x_{0}-x_{i_{k}}-x_{2 i_{k}}-\cdots-x_{\left(a_{1}-1\right) i_{k}} .
$$

In an analogous way we arrive by using (50) at the following (counterclockwise) order for the $\bar{x}_{i}, i=0, \ldots, a_{1}-1$ on the circle $S$ :

$$
\bar{x}_{0}-\bar{x}_{\left(a_{1}-1\right) i_{k}}-\bar{x}_{\left(a_{1}-2\right) i_{k}}-\cdots-\bar{x}_{i_{k}} \text {. }
$$

On $S$ we have

$$
\mathscr{L}\left(a_{S}\left(x_{i}, x_{i+l i_{k}}\right)\right)=2 \pi r l / a_{1}=\mathscr{L}\left(a_{S}\left(\bar{x}_{i}, \bar{x}_{i-l i_{k}}\right)\right),
$$

where $a_{S}(x, y)$ is the circular subarc of $S$ connecting $x$ and $y$ counterclockwise. Now we are going to determine the order on $S$ of both sets of points combined. To this extent, we consider a pair $\left(x_{j}, \bar{x}_{j}\right)$ such that $x_{j}$ minimizes dist $\left(x_{k}, v_{2} \cap S\right)$ for $k=0, \ldots, a_{1}-1$. Without loss of generality let this be $j=0$ and assume further without loss of generality that $a_{S}\left(x_{0}, \bar{x}_{0}\right) \leqslant a_{S}\left(\bar{x}_{0}, x_{0}\right)$. Now we claim

$$
\beta:=\mathscr{L}\left(a_{\mathrm{S}}\left(x_{0}, \bar{x}_{0}\right)\right)<2 \pi \mathrm{r} / \mathrm{a}_{1} .
$$

Indeed, if $\beta>2 \pi r / a_{1}$, then (53) implies $x_{i_{k}} \in a_{S}\left(x_{0}, \bar{x}_{0}\right)$ and therefore dist $\left(x_{i_{k}}, v_{2} \cap S\right)<$ $\operatorname{dist}\left(x_{0}, \nu_{2} \cap S\right)$, which contradicts the minimality of $x_{0}$. If $\beta=2 \pi r / a_{1}$, then $x_{i_{k}}=\bar{x}_{0}$, and for the lengths of the connecting subarcs on $\Gamma$ we have

$$
\mathrm{L} / 2=\left|s_{0}-\bar{s}_{0}\right|=\left|s_{0}-s_{i_{k}}\right|=s_{i_{k}}-s_{0} \stackrel{(48)}{=} \frac{L}{a_{1}} i_{k} .
$$

If $a_{1}$ is odd, this is a contradiction straight away. If $a_{1}$ is even, then $i_{k}=\frac{a_{1}}{2}>1$ since $a_{1} \geqslant 3$, and thus $\operatorname{gcd}\left(i_{k}, a_{1}\right)=i_{k}>1$. But recall that $i_{k}$ satisfies $k i_{k} \equiv a_{1} 1$, i.e., $k$ is the unique inverse to $i_{k}$ in $\mathbb{Z} / a_{1} \mathbb{Z}$, which exists if and only if $\operatorname{gcd}\left(i_{k}, a_{1}\right)=1$, contradiction. Therefore, our claim (54) is proven.

Combining (54) with (53) leads to the counterclockwise ordered combined chain

$$
x_{0}-\bar{x}_{0}-x_{i_{k}}-\bar{x}_{\left(a_{1}-1\right) i_{k}}-x_{2 i_{k}}-\bar{x}_{\left(a_{1}-2\right) i_{k}}-\cdots-x_{\left(a_{1}-1\right) i_{k}}-\bar{x}_{i_{k}},
$$

since there are no $x_{i}, \bar{x}_{i}$ in the circular arc $a_{S}\left(x_{0}, \bar{x}_{0}\right) \subset S$ because of the minimality of $x_{0}$, and the possible successors of $x_{0}$ and $\bar{x}_{0}$, respectively, are $x_{i_{k}}$ and $\bar{x}_{\left(a_{1}-1\right) i_{k}}$. Equation (53) delivers that $x_{i_{k}}$ has to appear before $\bar{x}_{\left(a_{1}-1\right) i_{k}}$. From there one can continue to form the whole combined chain (55).

The $a_{1}$-periodicity now gives us information on the shorter subarcs $a(p, q) \subset \Gamma$ connecting consecutive points $p$ and $q$ on the combined chain (55):

$$
a\left(x_{l i_{k}}, \bar{x}_{\left(a_{1}-l\right) i_{k}}\right)=\operatorname{Rot}\left(2 \pi l / a_{1}, v_{1}\right) a\left(x_{0}, \bar{x}_{0}\right) \text { for all } l \in \mathbb{N} .
$$

In particular, the lengths of these arcs coincide. But this leads to

$$
\mathscr{L}\left(a\left(x_{i_{k}}, \bar{x}_{i_{k}}\right)\right)=\left|s_{k_{k}}-\bar{s}_{i_{k}}\right|=L / 2=\left|s_{0}-\bar{s}_{0}\right|=\mathscr{L}\left(a\left(x_{0}, \bar{x}_{0}\right)\right)=\mathscr{L}\left(a\left(x_{i_{k}}, \bar{x}_{\left(a_{1}-1\right) i_{k}}\right)\right),
$$

and therefore $1=a_{1}-1$, which is not the case as $a_{1} \geqslant 3$. This final contradiction leads us to $v_{2} \cap \Gamma \neq \emptyset$. This establishes (1)(iii) and concludes the whole proof. 
Appendix A. Estimates for arClength PARAmetrizations

At the beginning of the proof of the second part of Theorem 3.2 we have used the following lemma stating that the (finite) Sobolev-Slobodetckij norm is conserved (up to constants) if one reparametrizes a regular absolutely continuous curve to arclength. Note that we have assumed $\alpha>2$ in that part of Theorem 3.2 , so that we state this auxiliary lemma in the range of Sobolev exponents that allow for a continuous embedding into classic function spaces with Hölder continuous first derivatives; cf. Remark 3.4 .

Lemma A.1. Assume that $\gamma \in W^{1+s, \rho}\left(\mathbb{R} / \mathbb{Z}, \mathbb{R}^{n}\right)$ for $\rho \in(1, \infty)$ and $s \in(1 / \rho, 1)$, and that $\left|\gamma^{\prime}\right|>0$ on $\mathbb{R} / \mathbb{Z}$. Then the corresponding arclength parametrization $\Gamma$ is of class $\mathrm{W}^{1+s, \rho}\left(\mathbb{R} /(\mathrm{LZ}), \mathbb{R}^{\mathrm{n}}\right)$ satisfying the estimate

$$
[\Gamma]_{W^{1+s, \rho}}^{\rho} \leqslant\left(\frac{1}{c}\right)^{1+(s+1) \rho}\left[1+\left(\frac{C}{c}\right)^{\rho}\right] C^{2} \cdot[\gamma]_{W^{1+s, \rho}}^{\rho},
$$

where $\mathrm{L}:=\mathscr{L}(\gamma)$ denotes the positive and finite length of $\gamma$, and $\mathrm{c}:=\min _{[0,1]}\left|\gamma^{\prime}\right|, \mathrm{C}:=$ $\max _{[0,1]}\left|\gamma^{\prime}\right|$.

Proof. Since $W^{1+s, \rho}\left(\mathbb{R} / \mathbb{Z}, \mathbb{R}^{n}\right)$ continuously embeds into $C^{1, s-(1 / \rho)}\left(\mathbb{R} / \mathbb{Z}, \mathbb{R}^{\mathfrak{n}}\right)$ we have

$$
c:=\min _{[0,1]}\left|\gamma^{\prime}\right| \leqslant\left|\gamma^{\prime}(\tau)\right| \leqslant \max _{[0,1]}\left|\gamma^{\prime}\right|=: C \text { for all } \tau \in[0,1],
$$

so that the arclength parameter $s(t):=\int_{0}^{t}\left|\gamma^{\prime}(\tau)\right| d \tau$ is a bi-Lipschitz continuous function $s:[0,1] \rightarrow[0, L]$ with

$$
c\left|t_{1}-t_{2}\right| \leqslant\left|s\left(t_{1}\right)-s\left(t_{2}\right)\right| \leqslant C\left|t_{1}-t_{2}\right| \text { for all } t_{1}, t_{2} \in[0,1],
$$

and its inverse function $t:=s^{-1}:[0, \mathrm{~L}] \rightarrow[0,1]$ satisfies

$$
\frac{1}{\mathrm{C}}\left|s_{1}-s_{2}\right| \leqslant\left|t\left(s_{1}\right)-\mathrm{t}\left(s_{2}\right)\right| \leqslant \frac{1}{\mathrm{c}}\left|s_{1}-s_{2}\right| \text { for all } s_{1}, s_{2} \in[0, \mathrm{~L}],
$$

Moreover, using (57) for the derivative $t^{\prime}(s)=1 /\left|\gamma^{\prime}(t(s))\right|$ one has

$$
\frac{1}{\mathrm{C}} \leqslant\left|\mathrm{t}^{\prime}(\mathrm{s})\right| \leqslant \frac{1}{\mathrm{c}} \text { for all } \mathrm{s} \in[0, \mathrm{~L}] .
$$

Now we start estimating the seminorm of the arclength parametrization $\Gamma(\cdot)=\gamma \circ \mathrm{t}(\cdot)$.

$$
\begin{aligned}
& {[\Gamma]_{W^{1+s, \rho}}^{\rho}=\int_{\mathbb{R} /(\mathrm{LZ})} \int_{-\mathrm{L} / 2}^{\mathrm{L} / 2} \frac{\left|\Gamma^{\prime}(\mathrm{u}+w)-\Gamma^{\prime}(\mathrm{u})\right|^{\rho}}{|w|^{1+s \rho}} \mathrm{d} w \mathrm{du}} \\
& =\int_{\mathbb{R} /(\mathrm{LZ})} \int_{-\mathrm{L} / 2}^{\mathrm{L} / 2} \frac{\left|\gamma^{\prime}(\mathrm{t}(\mathrm{u}+w)) \mathrm{t}^{\prime}(u+w)-\gamma^{\prime}(\mathrm{t}(\mathrm{u})) \mathrm{t}^{\prime}(u)\right|^{\rho}}{|\mathrm{t}(\mathrm{u}+w)-\mathrm{t}(\mathrm{u})|^{1+s \rho}} \cdot \frac{|\mathrm{t}(\mathrm{u}+w)-\mathrm{t}(\mathrm{u})|^{1+s \rho}}{|w|^{1+s \rho}} \mathrm{d} w \mathrm{~d} u \\
& \leqslant \int_{\mathbb{R} /(\mathrm{LZ})} \int_{-\mathrm{L} / 2}^{\mathrm{L} / 2} \frac{\left|\gamma^{\prime}(\mathrm{t}(\mathrm{u}+w))-\gamma^{\prime}(\mathrm{t}(\mathrm{u}))\right|^{\rho}\left|\mathrm{t}^{\prime}(\mathrm{u}+w)\right|^{\rho}}{|\mathrm{t}(\mathrm{u}+w)-\mathrm{t}(\mathrm{u})|^{1+s \rho}} \cdot \frac{|\mathrm{t}(\mathrm{u}+w)-\mathrm{t}(\mathrm{u})|^{1+s \rho}}{|w|^{1+s \rho}} \mathrm{d} w \mathrm{du} \\
& +\int_{\mathbb{R} /(\mathbb{Z})} \int_{-\mathrm{L} / 2}^{\mathrm{L} / 2} \frac{\left|\gamma^{\prime}(\mathrm{t}(\mathrm{u}))\right|^{\rho}\left|\mathrm{t}^{\prime}(\mathrm{u}+w)-\mathrm{t}^{\prime}(\mathrm{u})\right|^{\rho}}{|\mathrm{t}(\mathrm{u}+w)-\mathrm{t}(\mathrm{u})|^{1+s \rho}} \cdot \frac{|\mathrm{t}(\mathrm{u}+w)-\mathrm{t}(\mathrm{u})|^{1+s \rho}}{|w|^{1+s \rho}} \mathrm{d} w \mathrm{du} .
\end{aligned}
$$


By means of (60) and (59) we can estimate the first double integral on the right-hand side of (61) by

$$
\left(\frac{1}{c}\right)^{1+(s+1) \rho} \int_{\mathbb{R} /(\mathrm{LZ})} \int_{-\mathrm{L} / 2}^{\mathrm{L} / 2} \frac{\left|\gamma^{\prime}(\mathrm{t}(\mathrm{u}+w))-\gamma^{\prime}(\mathrm{t}(\mathrm{u}))\right|^{\rho}}{|\mathrm{t}(\mathrm{u}+w)-\mathrm{t}(\mathrm{u})|^{1+s \rho}} \mathrm{d} w \mathrm{~d} \mathrm{u} .
$$

With the help of (60) we find

$$
\left|\mathrm{t}^{\prime}(\mathrm{u}+w)-\mathrm{t}^{\prime}(\mathrm{u})\right|=\left|\frac{1}{\left|\gamma^{\prime}(\mathrm{t}(\mathrm{u}+w))\right|}-\frac{1}{\left|\gamma^{\prime}(\mathrm{t}(\mathrm{u}))\right|}\right| \stackrel{600}{\leqslant} \mathrm{c}^{-2}\left|\gamma^{\prime}(\mathrm{t}(\mathrm{u}))-\gamma^{\prime}(\mathrm{t}(\mathrm{u}+w))\right|,
$$

and this combined with (59) gives for the second double integral on the right-hand side of (61) the upper bound

$$
\left(\frac{C}{c^{2}}\right)^{\rho}\left(\frac{1}{c}\right)^{1+s \rho} \int_{\mathbb{R} /(L \mathbb{Z})} \int_{-L / 2}^{L / 2} \frac{\left|\gamma^{\prime}(t(u+w))-\gamma^{\prime}(t(u))\right|^{\rho}}{|t(u+w)-t(u)|^{1+s \rho}} d w d u .
$$

The integrals in (62) and (63) are identical and may be transformed using first the substitution $z(w):=\mathrm{t}(\mathrm{u}+w)$ with

$$
\mathrm{d} z(w)=\mathrm{t}^{\prime}(\mathrm{u}+w) \mathrm{d} w=\frac{1}{\left|\gamma^{\prime}(\mathrm{t}(\mathrm{u}+w))\right|} \mathrm{d} w=\frac{1}{\left|\gamma^{\prime}(z)\right|} \mathrm{d} w
$$

for the $w$-integration, giving

$$
\int_{\mathbb{R} /(\mathrm{LZ})} \int_{\mathfrak{t}(\mathfrak{u}-(\mathrm{L} / 2))}^{\mathfrak{t}(\mathfrak{u}+(\mathrm{L} / 2))}\left|\gamma^{\prime}(z)\right| \frac{\left|\gamma^{\prime}(z)-\gamma^{\prime}(\mathrm{t}(\mathrm{u}))\right|^{\rho}}{|z-\mathfrak{t}(\mathrm{u})|^{1+s \rho}} \mathrm{d} z \mathrm{du} .
$$

Due to the 1-periodicity the inner integral can be replaced by the integration over $\mathbb{R} / \mathbb{Z}$, and after applying Fubini's theorem we may change variables according to $y(u):=t(u)$ for the integration with respect to $u$ with $d y(u)=\left|\gamma^{\prime}(y)\right|^{-1} d u$, which by virtue of (57) leads to

$$
\int_{\mathbb{R} / \mathbb{Z}} \int_{\mathbb{R} / \mathbb{Z}}\left|\gamma^{\prime}(y)\right|\left|\gamma^{\prime}(z)\right| \frac{\left|\gamma^{\prime}(z)-\gamma^{\prime}(y)\right|^{\rho}}{|z-y|^{1+s \rho}} \stackrel{557}{\leqslant} C^{2}[\gamma]_{W^{1+s, \rho}}^{\rho} .
$$

Recall that (64) serves as an upper bound for the double integral that appears both in (62), and in (63). So, combining this with (61) leads to the desired estimate

$$
[\Gamma]_{W^{1+s, \rho}}^{\rho} \leqslant\left(\frac{1}{c}\right)^{1+(s+1) \rho}\left[1+\left(\frac{C}{c}\right)^{\rho}\right] C^{2} \cdot[\gamma]_{W^{1+s, \rho}}^{\rho} .
$$

With a simple argument (similar to the one in [31, Lemma 4.2]) we now show that injective curves parametrized by arclength of class $C^{1, \mu}$ are bi-Lipschitz.

Lemma A.2. Let $\mu \in(0,1], \mathrm{L}>0$, and $\Gamma \in \mathrm{C}^{1, \mu}\left(\mathbb{R} /(\mathrm{LZ}), \mathbb{R}^{\mathrm{n}}\right)$ with $\left|\Gamma^{\prime}\right| \equiv 1$ on $[0, \mathrm{~L}]$, such that $\left.\Gamma\right|_{[0, \mathrm{~L})}$ is injective. Then there is a constant $\mathrm{B}=\mathrm{B}(\mu, \Gamma) \geqslant 1$ such that

$$
\frac{1}{\mathrm{~B}}|w| \leqslant|\Gamma(u+w)-\Gamma(u)| \leqslant|w| \text { for all } u \in \mathbb{R} /(\mathrm{L} \mathbb{Z}),|w| \leqslant \mathrm{L} / 2 .
$$

From the Morrey-type embedding mentioned in Remark 3.4 and the specification in (11) we directly derive the following corollary. 
Corollary A.3. Let $\mathrm{L}>0, \rho \in(1, \infty), s \in(1 / \rho, 1)$, and $\Gamma \in \mathrm{W}^{1+s, \rho}\left(\mathbb{R} /(\mathrm{L} \mathbb{Z}), \mathbb{R}^{\mathrm{n}}\right)$ be an injective arclength parametrized curve. Then there is a constant $\mathrm{B}=\mathrm{B}(\mathrm{s}, \rho, \Gamma) \geqslant 1$ such that

$$
\frac{1}{\mathrm{~B}}|w| \leqslant|\Gamma(u+w)-\Gamma(u)| \leqslant|w| \text { for all } u \in \mathbb{R} /(\mathrm{L} \mathbb{Z}),|w| \leqslant \mathrm{L} / 2 .
$$

In particular, there is a constant $\mathrm{B}=\mathrm{B}(\alpha, \Gamma)$ such that any injective arclength parametrized curve $\Gamma \in \mathrm{W}^{(\alpha+1) / 2,2}\left(\mathbb{R} /(\mathrm{LZ}), \mathbb{R}^{n}\right)$ satisfies (65).

Proof of Lemma A.2 We only need to prove the left inequality of the bi-Lipschitz estimate since the upper bound follows from $\left|\Gamma^{\prime}\right| \equiv 1$ on $[0, \mathrm{~L}]$. Without loss of generality we may assume that $\Gamma^{\prime}(u)=(1,0 \ldots, 0) \in \mathbb{R}^{n}$ so that we may estimate the tangent's first component $\Gamma_{1}^{\prime}$ from below as

$$
\begin{aligned}
\Gamma_{1}^{\prime}(u+w) & \geqslant \Gamma_{1}^{\prime}(u)-\left|\Gamma_{1}^{\prime}(u)-\Gamma_{1}^{\prime}(u+w)\right| \\
& \geqslant 1-\|\Gamma\|_{C^{1, \mu}}|w|^{\mu} \geqslant \frac{3}{4} \quad \text { for all }|w| \leqslant \varepsilon_{0}:=\left(\frac{1}{4\|\Gamma\|_{C^{1, \mu}}}\right)^{1 / \mu},
\end{aligned}
$$

which implies

$$
|\Gamma(u+w)-\Gamma(u)| \geqslant\left|\Gamma_{1}(u+w)-\Gamma_{1}(u)\right|=\left|\int_{u}^{u+w} \Gamma_{1}^{\prime}(\tau) d \tau\right| \geqslant \frac{3}{4}|w| \text { for all }|w| \leqslant \varepsilon_{0} .
$$

The continuous function $\mathrm{g}(\mathrm{u}, \mathrm{w}):=|\Gamma(\mathrm{u}+\mathrm{w})-\Gamma(\mathrm{u})|$, on the other hand, is uniformly continuous on the compact set

$$
\Sigma:=\left\{(\mathrm{u}, w) \in \mathbb{R} /(\mathrm{L} \mathbb{Z}) \times[-\mathrm{L} / 2, \mathrm{~L} / 2]:|w| \geqslant \varepsilon_{0}\right\},
$$

and $g$ is strictly positive on $\Sigma$ since $\Gamma_{[0, \mathrm{~L})}$ is assumed to be injective. Hence there is a positive constant $\mathrm{c}=\mathrm{c}(\Gamma)$ such that $\left.\mathrm{g}\right|_{\Sigma} \geqslant \mathrm{c}$, which implies

$$
|\Gamma(u+w)-\Gamma(u)| \geqslant c \geqslant \frac{2 c}{L}|w| \text { for all } \varepsilon_{0} \leqslant|w| \leqslant L / 2 .
$$

Combining (66) with (67) we obtain the desired bi-Lipschitz estimate for the constant $\mathrm{B}=\mathrm{B}(\mu, \Gamma):=\max \left\{\frac{4}{3}, \frac{\mathrm{L}}{2 \mathrm{c}}\right\}$.

In the proof of part (ii) of Theorem 3.2 we have also used the following elementary inequality.

Lemma A.4. For any $\alpha \in(1, \infty)$ one has

$$
1-\chi^{\alpha} \leqslant(\alpha+1)(1-x) \text { for all } x \in[0,1] .
$$

In particular, if $\alpha \in[2, \infty)$, the following holds.

$$
1-x^{\alpha} \leqslant(\alpha+1)\left(1-x^{2}\right) \text { for all } x \in[0,1] .
$$

Proof. It suffices to prove that the function $f_{\alpha}(x):=x^{\alpha}-(\alpha+1) \chi+\alpha$ is non-negative for all $x \in[0,1]$, and for all $\alpha \in(1, \infty)$, since $f$ may be rewritten as

$$
f(x)=x^{\alpha}+(\alpha+1)(1-x)-1 \text {. }
$$

One immediately checks for the derivative (which exists as $\alpha>1$ )

$$
f^{\prime}(x)=\alpha x^{\alpha-1}-(\alpha+1) \leqslant-1 \text { for all } x \in[0,1],
$$

so that $f$ strictly decreases from the positive value $f(0)=\alpha$ to the value $f(1)=0$ on $[0,1]$. 
Lemma 4.8 requires the existence of some $k \in \mathbb{Z}$ satisfying specific equivalence class relations, established in the following elementary result.

Lemma A.5. For relatively prime numbers $a, b \in \mathbb{Z} \backslash\{0, \pm 1\}$ and some $m \in \mathbb{N}, m>1$, dividing either a or $\mathrm{b}$, there is an integer $\mathrm{k} \in \mathbb{Z}$, which is unique modulo $\mathrm{m}$, such that

$$
\begin{cases}{[a k+1]=e=[0] \in \mathbb{Z} / m \mathbb{Z}} & \text { if } m \mid b \\ {[b k+1]=e=[0] \in \mathbb{Z} / m \mathbb{Z}} & \text { if } m \mid a\end{cases}
$$

Proof. It suffices to treat the case $m \mid b$. The required condition $[a k+1]=[0]$ (identifying $k$ uniquely modulo $\mathrm{m})$ is equivalent to $[a k]=[-1]$ or $[(-a) k]=[1]$, which means that $(-a)$ is invertible modulo $m$, or, equivalently that $(-a)$ and $m$ are relatively prime. Assuming that there is a common divisor $d \in \mathbb{Z},|d| \geqslant 2$ of $(-a)$ and $m$, then $d$ divides also $b$ since $\mathrm{m} \mid \mathrm{b}$, but this contradicts our assumption that $\mathrm{a}$ and $\mathrm{b}$ are relatively prime.

For the proof of Theorem 1.2 we needed the following elementary number theoretical result.

Lemma A.6. If two integers $\mathrm{a}, \mathrm{b} \in \mathbb{Z} \backslash\{0\}$ are relatively prime then also the two integers $\mathrm{a}+\mathrm{b}$ and $\mathrm{ab}$.

Proof. Let $\operatorname{gcd}(a, b)=1$. Assuming that $a+b$ and $a b$ are not relatively prime, we can find a prime $n$ such that $n \mid(a+b)$ and $n \mid a b$. The second condition implies $n \mid a$ or $n \mid b$. Without loss of generality we assume $n \mid a$, as for $n \mid b$ the argumentation is analogous. Combining $n \mid a$ with $n \mid(a+b)$, we arrive at $n \mid b$, which contradicts $\operatorname{gcd}(a, b)=1$.

In the proof of Theorem 4.12 we used the following simple result concerning images of rotationally symmetric sets under isometries of $\mathbb{R}^{3}$.

Lemma A.7. Let $v \in \mathbb{S}^{2}, \beta \in \mathbb{R}$, and $\mathrm{I}: \mathbb{R}^{3} \rightarrow \mathbb{R}^{3}$ an orientation preserving isometry of $\mathbb{R}^{3}$ with $\mathrm{I}(v) \neq 0$. Then for any set $M \subset \mathbb{R}^{3}$ with

$$
\operatorname{Rot}(\beta, \mathbb{R} v) M=M
$$

one has

$$
\operatorname{Rot}(\beta, I(\mathbb{R} v)) I(M)=I(M),
$$

where similarly as before $\operatorname{Rot}(\beta, w)$ stands for the rotation about the affine line $w=\mathbb{R e}_{w}+$ $\mathrm{d} \subset \mathbb{R}^{3}$ for some $e_{w} \in \mathbb{S}^{2}$ and $\mathrm{d} \in \mathbb{R}^{3}$ with rotational angle $\beta \in \mathbb{R}$. (For $\beta>0$ with $\beta \notin \pi \mathbb{Z}$ and any $\xi \notin \mathcal{w}$, the set

$$
\mathscr{B}:=\left\{\xi-\Pi_{w}(\xi), \operatorname{Rot}(\beta, w) \xi-\Pi_{w}(\operatorname{Rot}(\beta, w) \xi), e_{w}\right\}
$$

forms a positively oriented basis of $\mathbb{R}^{3}$. Here $\Pi_{w}$ denotes the orthogonal projection onto the affine line $w$.

Proof. The statement is trivial for $\beta=0$ since the rotations involved are simply the identity mapping in $\mathbb{R}^{3}$. Without loss of generality let $\beta>0$. For $Y \in I(M)$ there is exactly one $\eta \in M$ such that $Y=I(\eta)$. Exploiting assumption (69) we find exactly one $\xi \in M$ such that

\footnotetext{
${ }^{4}$ That is, the $3 \times 3$-matrix mapping $\mathscr{B}$ onto the standard basis $\left\{e_{1}, e_{2}, e_{3}\right\}$ has positive determinant.
} 
$\eta=\operatorname{Rot}(\beta, \mathbb{R} v) \xi$. Let $\xi_{0}:=\Pi_{\mathbb{R} v}(\eta)$ be the orthogonal projection of $\eta$ onto the rotational axis $\mathbb{R} v$ so that

$$
\xi_{0} \in \mathbb{R} v, \quad\left(\xi-\xi_{0}\right) \perp v, \quad\left(\eta-\xi_{0}\right) \perp v, \quad\left|\xi-\xi_{0}\right|=\left|\eta-\xi_{0}\right|
$$

and such that the set $\mathscr{C}:=\left\{\xi-\xi_{0}, \eta-\xi_{0}, v\right\}$ forms a positively oriented basis of $\mathbb{R}^{3}$ if $\eta \notin \mathbb{R} v$ and $\beta \notin \pi \mathbb{Z}$. Since $I$ is an orientation preserving isometry we can write $I x=S x+b, x \in \mathbb{R}^{3}$, for some $S \in S O(3)$ and $b \in \mathbb{R}^{3}$, and find $\mathrm{I}\left(\xi_{0}\right) \in \mathrm{I}(\mathbb{R} v)$ and

$$
\left(\mathrm{I}(\xi)-\mathrm{I}\left(\xi_{0}\right)\right) \perp \mathrm{S} v, \quad\left(\mathrm{I}(\eta)-\mathrm{I}\left(\xi_{0}\right)\right) \perp S v, \quad\left|\mathrm{I}(\xi)-\mathrm{I}\left(\xi_{0}\right)\right|=\left|\mathrm{I}(\eta)-\mathrm{I}\left(\xi_{0}\right)\right|,
$$

and the set $\mathscr{D}:=\left\{\mathrm{I}(\xi)-\mathrm{I}\left(\xi_{0}\right), \mathrm{I}(\eta)-\mathrm{I}\left(\xi_{0}\right), S v\right\}$ forms a positively oriented basis of $\mathbb{R}^{3}$. In addition, by isometry,

$$
\cos \beta=\frac{\left(\xi-\xi_{0}\right) \cdot\left(\eta-\xi_{0}\right)}{\left|\xi-\xi_{0}\right|\left|\eta-\xi_{0}\right|}=\frac{\left(\mathrm{I}(\xi)-\mathrm{I}\left(\xi_{0}\right)\right) \cdot\left(\mathrm{I}(\eta)-\mathrm{I}\left(\xi_{0}\right)\right)}{\left|\mathrm{I}(\xi)-\mathrm{I}\left(\xi_{0}\right)\right|\left|\mathrm{I}(\eta)-\mathrm{I}\left(\xi_{0}\right)\right|},
$$

which is also true for $\beta \in \pi \mathbb{Z}$, so that for $X:=I(\xi)$ we arrive at

$$
\operatorname{Rot}(\beta, I(\mathbb{R} v)) X=\operatorname{Rot}(\beta, I(\mathbb{R} v)) I(\xi)=I(\eta)=Y,
$$

which proves the inclusion

$$
\mathrm{I}(M) \subset \operatorname{Rot}(\beta, I(\mathbb{R} v)) \mathrm{I}(M) \quad \text { for arbitrary } \beta \in \mathbb{R} \backslash\{0\},
$$

since the same argument works for $\beta<0$ only with negatively oriented bases $\mathscr{C}$ and $\mathscr{D}$. The inclusion (71) is trivial if $Y=I(\eta)$ for some $\eta \in \mathbb{R} v$ because then $\operatorname{Rot}(\beta, I(\mathbb{R} v)) \mathrm{I}(\eta)=$ $\mathrm{I}(\eta)$. Since we proved (71) for arbitrary $\beta \neq 0$ we can apply the inverse rotation $\operatorname{Rot}(\beta, \mathrm{I}(\mathbb{R} v))^{-1}=$ $\operatorname{Rot}(-\beta, I(\mathbb{R} v))$ to $(71)$ and use the above argument again.

Lemma A.8. Let $\mathrm{A} \in \mathrm{SO}(3)$ be a rotational matrix with angle $\phi=2 \pi / \mathrm{b}, \mathrm{b} \in \mathbb{N}$, about the z-axis and $M \subset \mathbb{R}^{3}$ be a set invariant with respect to said rotation, i.e. $A M=M$. For any rotational matrix $\mathrm{B} \in \mathrm{SO}(3)$ about an axis $v$ with $v \perp e_{3}, v \cap \mathbb{R} e_{3}=\{0\}$, and rotational angle $\pi$ we have

$$
\mathrm{ABM}=\mathrm{BAM}=\mathrm{BM} .
$$

Proof. The case $b=1$ is trivial. Therefore let $\phi=2 \pi / b, b \geqslant 2$, be the rotational angle of $A$, and $e_{v} \in \mathbb{S}^{2}$ be a unit vector contained in $v$, and set $f:=e_{3} \wedge e_{v}$. The matrix representations of $A$ and $B$ with respect to the orthonormal basis $\mathscr{B}:=\left\{e_{v}, f, e_{3}\right\}$ are given by

$$
\mathrm{A}=\left(\begin{array}{ccc}
\cos \phi & -\sin \phi & 0 \\
\sin \phi & \cos \phi & 0 \\
0 & 0 & 1
\end{array}\right), \quad \mathrm{B}=\left(\begin{array}{ccc}
1 & 0 & 0 \\
0 & -1 & 0 \\
0 & 0 & -1
\end{array}\right)
$$

Further, the assumption $A M=M$ implies

$$
y:=A^{k} x \in M \text { for all } x \in M, k \in \mathbb{Z} /(b \mathbb{Z}) .
$$

Hence it suffices to show that there is $k \in \mathbb{Z} /(b \mathbb{Z})$ such that

$$
A B x=B A A^{k} x \text { for all } x \in M
$$

to prove the inclusion $A B M \subset B A M$. On the other hand, if (72) is established for some $k \in \mathbb{Z} /(b \mathbb{Z})$ then we can use our assumption $A M=M$, hence also $A^{k} M=M$ again to write any $y \in M$ as $A^{k} x=y$ for an appropriate $x \in M$, so that (72) implies also the reverse inclusion $B A M \subset A B M$. 
To establish (72) we calculate for $x=\left(x^{1}, x^{2}, x^{3}\right) \in M$

$$
\begin{aligned}
\mathrm{AB} x & =\left(\begin{array}{ccc}
\cos \phi & -\sin \phi & 0 \\
\sin \phi & \cos \phi & 0 \\
0 & 0 & 1
\end{array}\right)\left(\begin{array}{ccc}
1 & 0 & 0 \\
0 & -1 & 0 \\
0 & 0 & -1
\end{array}\right) \chi=\left(\begin{array}{ccc}
\cos \phi & -\sin \phi & 0 \\
\sin \phi & \cos \phi & 0 \\
0 & 0 & 1
\end{array}\right)\left(\begin{array}{c}
\chi^{1} \\
-\chi^{2} \\
-\chi^{3}
\end{array}\right) \\
& =\left(\begin{array}{c}
\chi^{1} \cos (2 \pi / b)+\chi^{2} \sin (2 \pi / b) \\
x^{1} \sin (2 \pi / b)-\chi^{2} \cos (2 \pi / b) \\
-\chi^{3}
\end{array}\right)
\end{aligned}
$$

as well as

$$
\begin{aligned}
\mathrm{BAA}^{k} \chi=\mathrm{BA}^{\mathrm{k}+1} \chi & =\left(\begin{array}{ccc}
1 & 0 & 0 \\
0 & -1 & 0 \\
0 & 0 & -1
\end{array}\right)\left(\begin{array}{ccc}
\cos \phi & -\sin \phi & 0 \\
\sin \phi & \cos \phi & 0 \\
0 & 0 & 1
\end{array}\right)^{\mathrm{k}+1}\left(\begin{array}{l}
x^{1} \\
x^{2} \\
\chi^{3}
\end{array}\right) \\
& =\left(\begin{array}{ccc}
1 & 0 & 0 \\
0 & -1 & 0 \\
0 & 0 & -1
\end{array}\right)\left(\begin{array}{ccc}
\cos ((k+1) \phi) & -\sin ((k+1) \phi) & 0 \\
\sin ((k+1) \phi) & \cos ((k+1) \phi) & 0 \\
0 & 0 & 1
\end{array}\right)\left(\begin{array}{l}
x^{1} \\
x^{2} \\
x^{3}
\end{array}\right) \\
& =\left(\begin{array}{c}
x^{1} \cos (2 \pi(k+1) / b)-\chi^{2} \sin (2 \pi(k+1) / b) \\
-x^{1} \sin (2 \pi(k+1) / b)-x^{2} \cos (2 \pi(k+1) / b) \\
-\chi^{3}
\end{array}\right)
\end{aligned}
$$

Due to the symmetry properties of sine and cosine we arrive at (72) if and only if $k+1 \equiv_{\mathrm{b}}$ -1 or $k=-2 \bmod b$.

\section{Acknowledgments}

Part of this work is contained in the first author's Ph.D. thesis [16]. The second author's work is partially funded by DFG Grant no. Mo 966/7-1 Geometric curvature functionals: energy landscape and discrete methods and by the Excellence Initiative of the German federal and state governments.

\section{REFERENCES}

[1] Abrams, A., Cantarella, J., Fu, J. H. G., Ghomi, M., and Howard, R. Circles minimize most knot energies. Topology 42, 2 (2003), 381-394.

[2] Bergh, J., ANd Löfström, J. Interpolation spaces. An introduction. Springer-Verlag, Berlin-New York, 1976. Grundlehren der Mathematischen Wissenschaften, No. 223.

[3] Blatt, S. Note on continuously differentiable isotopies. Report 34, RWTH Aachen University, 2009.

[4] Blatt, S. Boundedness and regularizing effects of O'Hara's knot energies. J. Knot Theory Ramifications 21, 1 (2012), 1250010, 9.

[5] Blatt, S. The gradient flow of O'Hara's knot energies. Math. Ann. 370, 3-4 (2018), 993-1061.

[6] Blatt, S., Gilsbach, A., And von der Mosel, H. Symmetric Möbius critical knots, 2018. Preprint in preparation.

[7] Blatt, S., ANd Reiter, P. Stationary points of O’Hara's knot energies. Manuscripta Math. 140, 1-2 (2013), 29-50.

[8] Brakke, K. A. The surface evolver. Experiment. Math. 1, 2 (1992), 141-165.

[9] Burde, G., And Zieschang, H. Knots, vol. 5 of De Gruyter Studies in Mathematics. Walter de Gruyter \& Co., Berlin, 1985.

[10] Cantarella, J., Fu, J. H. G., Mastin, M., and Ellis Royal, J. Symmetric criticality for tight knots. J. Knot Theory Ramifications 23, 2 (2014), 1450008, 17. 
[11] Conn, P. M. Lie groups. Cambridge Tracts in Mathematics and Mathematical Physics, no. 46. Cambridge University Press, New York, N.Y., 1957.

[12] Crowell, R. H., ANd Fox, R. H. Introduction to knot theory. Springer-Verlag, New York-Heidelberg, 1977. Reprint of the 1963 original, Graduate Texts in Mathematics, No. 57.

[13] Di Nezza, E., Palatucci, G., and Valdinoci, E. Hitchhiker's guide to the fractional Sobolev spaces. Bull. Sci. Math. 136, 5 (2012), 521-573.

[14] Fischer, G. Lehrbuch der Algebra. Friedr. Vieweg \& Sohn Verlag, Wiesbaden (2013).

[15] Freedman, M. H., He, Z.-X., And Wang, Z. Möbius energy of knots and unknots. Ann. of Math. (2) 139, 1 (1994), 1-50.

[16] GILsBach, A. On symmetric critical points of knot energies. PhD thesis, RWTH Aachen University, 2018. http://publications.rwth-aachen.de/record/726186/files/726186.pdf.

[17] Gilsbach, A., Knappmann, J., and von der Mosel, H. Many knot energies possess at least two critical points in every torus knot class, 2018. Preprint in preparation.

[18] Grünbaum, B., And Shephard, G. Patterns on the 2-sphere. Mathematika 28, 1 (1981), 1-35.

[19] Grünbaum, B., and Shephard, G. Symmetry groups of knots. Mathematics Magazine 58, 3 (1985), 161165.

[20] Hermes, T. Analysis of the first variation and a numerical gradient flow for integral Menger curvature. ArXiv e-prints (Aug. 2014).

[21] Kaufrman, L. H. Following knots down their energy gradients. Symmetry 4, 2 (2012), 276-284.

[22] Kim, D., And Kusner, R. Torus knots extremizing the Möbius energy. Experiment. Math. 2, 1 (1993), $1-9$.

[23] Livingston, C. Knotentheorie für Einsteiger. Wiesbaden: Vieweg, 1995.

[24] Nitsche, J. C. C. The smallest sphere containing a rectifiable curve. Amer. Math. Monthly 78 (1971), 881-882.

[25] O'Hara, J. Family of energy functionals of knots. Topology Appl. 48, 2 (1992), 147-161.

[26] O'Hara, J. Energy functionals of knots. II. Topology Appl. 56, 1 (1994), 45-61.

[27] Palais, R. S. The principle of symmetric criticality. Comm. Math. Phys. 69, 1 (1979), 19-30.

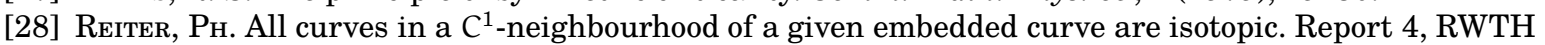
Aachen University, 2005.

[29] Runst, T., And Sickel, W. Sobolev spaces of fractional order, Nemytskij operators, and nonlinear partial differential equations, vol. 3 of De Gruyter Series in Nonlinear Analysis and Applications. Walter de Gruyter \& Co., Berlin, 1996.

[30] Scharein, R. KnotPlot, 2017. Program for drawing, visualizing, manipulating, and energy minimizing knots. Version from March 10, 2017. See http://www.knotplot.com.

[31] Strzelecki, P., Szumańska, M., And von der Mosel, H. A geometric curvature double integral of Menger type for space curves. Ann. Acad. Sci. Fenn. Math. 34, 1 (2009), 195-214.

[32] Strzelecki, P., Szumańska, M., and von der Mosel, H. Regularizing and self-avoidance effects of integral Menger curvature. Ann. Sc. Norm. Super. Pisa Cl. Sci. (5) 9, 1 (2010), 145-187.

[33] Strzelecki, P., Szumańska, M., and von der Mosel, H. On some knot energies involving Menger curvature. Topology Appl. 160, 13 (2013), 1507-1529.

[34] Strzelecki, P., ANd von der Mosel, H. Tangent-point self-avoidance energies for curves. J. Knot Theory Ramifications 21, 5 (2012), 1250044, 28.

[35] Triebel, H. Theory of function spaces, vol. 38 of Mathematik und ihre Anwendungen in Physik und Technik [Mathematics and its Applications in Physics and Technology]. Akademische Verlagsgesellschaft Geest \& Portig K.-G., Leipzig, 1983.

[36] Triebel, H. Theory of function spaces. II, vol. 84 of Monographs in Mathematics. Birkhäuser Verlag, Basel, 1992.

[37] Triebel, H. Theory of function spaces. III, vol. 100 of Monographs in Mathematics. Birkhäuser Verlag, Basel, 2006. 
(A. Gilsbach)

INSTITUT FÜr Mathematik

RWTH AaChEN UnIVERSITY

TEMPLERGRABEN 55

D-52062 Aachen, Germany

E-mail address: gilsbach@instmath.rwth-aachen.de

(H. von der Mosel)

Institut Für Mathematik

RWTH AACHEN UNIVERSITY

Templergraben 55

D-52062 Aachen, Germany

E-mail address: heiko@instmath.rwth-aachen.de 